\title{
Environmental Noise in Advanced LIGO Detectors
}

P Nguyen ${ }^{1}$, R M S Schofield ${ }^{1}$, A Effler ${ }^{2}$, C Austin ${ }^{3}$, V Adya ${ }^{4}$, M Ball ${ }^{1}$, S Banagiri ${ }^{5}$, K Banowetz ${ }^{6}$, C Billman ${ }^{7}$, C D Blair ${ }^{8,2}$, A Buikema ${ }^{9,2}$, C Cahillane ${ }^{6,10}$, F Clara ${ }^{10}$, P B Covas ${ }^{11}$, G Dalya ${ }^{12}$, C Daniel ${ }^{6}$, B Dawes ${ }^{6}$, R DeRosa ${ }^{2}$, S E Dwyer ${ }^{10}$, R Frey ${ }^{1}$, V V Frolov ${ }^{2}$, D Ghirado ${ }^{6}$, E Goetz ${ }^{3,13,10}$, T Hardwick ${ }^{3}$, A F Helmling-Cornell ${ }^{1}$, I J Hollows ${ }^{14}$, N Kijbunchoo ${ }^{15}$,

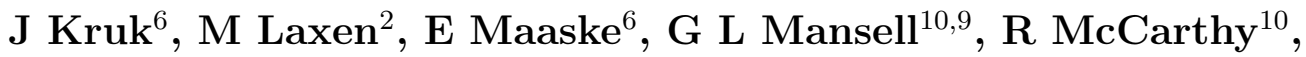
K Merfeld ${ }^{1}$, A Neunzert ${ }^{16}$, J R Palamos ${ }^{1}$, W Parker ${ }^{2,17}$, B Pearlstone ${ }^{18}$, A Pele ${ }^{2}$, H Radkins ${ }^{10}$, V Roma ${ }^{1}$, R L Savage ${ }^{10}$, P Schale ${ }^{1}$, D Shoemaker ${ }^{9}$, T Shoemaker $^{6}$, S Soni $^{3}$, D Talukder ${ }^{1}$, M Tse ${ }^{9}$, G Valdes ${ }^{3}$, M Vidreo ${ }^{6}$, C Vorvick $^{10}$, R Abbott ${ }^{6}$, C Adams ${ }^{2}$, R X Adhikari ${ }^{6}$, A Ananyeva ${ }^{6}$, S Appert ${ }^{6}, \mathrm{~K}$ Arai $^{6}$, J S Areeda ${ }^{19}$, Y Asali ${ }^{20}$, S M Aston ${ }^{2}$, A M Baer ${ }^{21}$,

S W Ballmer ${ }^{22}$, D Barker ${ }^{10}$, L Barsotti ${ }^{9}$, J Bartlett ${ }^{10}$, B K Berger ${ }^{23}$, J Betzwieser ${ }^{2}$, D Bhattacharjee ${ }^{13}$, G Billingsley ${ }^{6}$, S Biscans ${ }^{9,6}$, R M Blair ${ }^{10}, \mathbf{N}$ Bode $^{4,24}, \mathbf{P}$ Booker $^{4,24}, \mathbf{R}$ Bork $^{6}$, A Bramley ${ }^{2}$, A F Brooks ${ }^{6}$, D D Brown ${ }^{25}$, K C Cannon ${ }^{26}$, X Chen ${ }^{8}$, A A Ciobanu ${ }^{25}$, S J Cooper ${ }^{27}$, C M Compton ${ }^{10}$, K R Corley ${ }^{20}$, S T Countryman ${ }^{20}$, D C Coyne ${ }^{6}$, L E H Datrier ${ }^{18}$, D Davis ${ }^{22}$, C Di Fronzo ${ }^{27}$, K L Dooley ${ }^{28,29}$, J C Driggers ${ }^{10}$, P Dupej ${ }^{18}$, T Etzel ${ }^{6}$, M Evans ${ }^{9}$, T M Evans ${ }^{2}$, J Feicht ${ }^{6}$, A Fernandez-Galiana ${ }^{9}$, P Fritschel ${ }^{9}$, P Fulda $^{7}$, M Fyffe ${ }^{2}$, J A Giaime ${ }^{3,2}$, K D Giardina ${ }^{2}$, P Godwin ${ }^{30}$, S Gras ${ }^{9}$, C Gray ${ }^{10}$, R Gray ${ }^{18}$, A C Green ${ }^{7}$, E K Gustafson ${ }^{6}$, R Gustafson ${ }^{16}$, J Hanks ${ }^{10}$, J Hanson ${ }^{2}$, R K Hasskew ${ }^{2}$, M C Heintze ${ }^{2}$, N A Holland ${ }^{15}$, J D Jones ${ }^{10}$, S Kandhasamy ${ }^{31}$, S Karki ${ }^{1}$, M Kasprzack ${ }^{6}$, K Kawabe ${ }^{10}$, P J King ${ }^{10}$, J S Kissel ${ }^{10}$, Rahul Kumar ${ }^{10}$, M Landry ${ }^{10}$, B B Lane ${ }^{9}$, B Lantz ${ }^{23}$, Y K Lecoeuche ${ }^{10}$, J Leviton ${ }^{16}$, J Liu ${ }^{4,24}$, M Lormand ${ }^{2}$, A P Lundgren ${ }^{32}$, R Macas ${ }^{28}, \mathrm{M}$ MacInnis $^{9}$, D M Macleod ${ }^{28}$, S Márka ${ }^{20}$, Z Márka ${ }^{20}$, D V Martynov ${ }^{27}, \mathrm{~K} \mathrm{Mason}^{9}$, T J Massinger ${ }^{9}$, F Matichard ${ }^{6,9}$, N Mavalvala ${ }^{9}$, D E McClelland ${ }^{15}$, S McCormick ${ }^{2}$, L McCuller ${ }^{9}$, J McIver ${ }^{6}$, T McRae ${ }^{15}$, G Mendell ${ }^{10}$, E L Merilh ${ }^{10}$, F Meylahn ${ }^{4,24}$, P M Meyers ${ }^{33}$, T Mistry ${ }^{14}$, R Mittleman ${ }^{9}$, G Moreno ${ }^{10}$, C M Mow-Lowry ${ }^{27}$, S Mozzon ${ }^{32}$, A Mullavey ${ }^{2}$, T J N Nelson ${ }^{2}$, 
L K Nuttall ${ }^{32}$, J Oberling ${ }^{10}$, Richard J Oram ${ }^{2}$, C Osthelder ${ }^{6}$, D J Ottaway ${ }^{25}$, H Overmier ${ }^{2}$, E Payne ${ }^{34}$, R Penhorwood ${ }^{16}$, C J Perez ${ }^{10}$, M Pirello ${ }^{10}$, K E Ramirez ${ }^{35}$, J W Richardson ${ }^{6}$, K Riles ${ }^{16}$, N A Robertson ${ }^{6,18}$, J G Rollins ${ }^{6}$, C L Romel ${ }^{10}$, J H Romie ${ }^{2}$, M P Ross ${ }^{36}$, K Ryan ${ }^{10}$, T Sadecki ${ }^{10}$, E J Sanchez ${ }^{6}$, L E Sanchez ${ }^{6}, \mathbf{T}$ R Saravanan ${ }^{31}$, D Schaetzl ${ }^{6}, \mathbf{R}$ Schnabel $^{37}$, E Schwartz ${ }^{2}$, D Sellers ${ }^{2}$, T Shaffer ${ }^{10}$, D Sigg $^{10}$, B J J Slagmolen ${ }^{15}$, J R Smith ${ }^{19}$, B Sorazu ${ }^{18}$, A P Spencer ${ }^{18}$, K A Strain ${ }^{18}$, L Sun ${ }^{6}$, M J Szczepańczyk ${ }^{7}, \mathbf{M}$ Thomas $^{2}$,

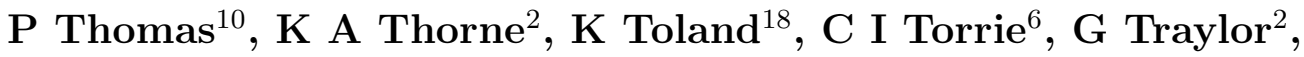
A L Urban ${ }^{3}$, G Vajente ${ }^{6}$, D C Vander-Hyde ${ }^{22}$, P J Veitch ${ }^{25}$, K Venkateswara ${ }^{36}$, G Venugopalan ${ }^{6}$, A D Viets ${ }^{38}, \mathbf{T}$ Vo $^{22}$, M Wade ${ }^{39}$, R L Ward ${ }^{15}$, J Warner ${ }^{10}$, B Weaver ${ }^{10}, \mathbf{R}$ Weiss $^{9}$, C Whittle ${ }^{9}$, B Willke ${ }^{24,4}$, C C Wipf ${ }^{6}$, L Xiao $^{6}, \mathbf{H}$ Yamamoto $^{6}$, Hang $\mathrm{Yu}^{9}$, Haocun $\mathrm{Yu}^{9}$, L Zhang ${ }^{6}, \mathrm{M}$ E Zucker ${ }^{9,6}$, and J Zweizig ${ }^{6}$

${ }^{1}$ University of Oregon, Eugene, OR 97403, USA

${ }^{2}$ LIGO Livingston Observatory, Livingston, LA 70754, USA

${ }^{3}$ Louisiana State University, Baton Rouge, LA 70803, USA

${ }^{4}$ Max Planck Institute for Gravitational Physics (Albert Einstein Institute), D-30167

Hannover, Germany

${ }^{5}$ University of Minnesota, Minneapolis, MN 55455, USA

${ }^{6}$ LIGO, California Institute of Technology, Pasadena, CA 91125, USA

${ }^{7}$ University of Florida, Gainesville, FL 32611, USA

${ }^{8}$ OzGrav, University of Western Australia, Crawley, Western Australia 6009, Australia

${ }^{9}$ LIGO, Massachusetts Institute of Technology, Cambridge, MA 02139, USA

${ }^{10}$ LIGO Hanford Observatory, Richland, WA 99352, USA

${ }^{11}$ Universitat de les Illes Balears, IAC3-IEEC, E-07122 Palma de Mallorca, Spain

${ }^{12}$ Eötvös Loránd University, H-1053 Budapest, Egyetem tér 1-3, 1053 Hungary

${ }^{13}$ Missouri University of Science and Technology, Rolla, MO 65409, USA

${ }^{14}$ The University of Sheffield, Sheffield S10 2TN, UK

${ }^{15}$ OzGrav, Australian National University, Canberra, Australian Capital Territory 0200, Australia

${ }^{16}$ University of Michigan, Ann Arbor, MI 48109, USA

${ }^{17}$ Southern University and A\&M College, Baton Rouge, LA 70813, USA

${ }^{18}$ SUPA, University of Glasgow, Glasgow G12 8QQ, UK

${ }^{19}$ California State University Fullerton, Fullerton, CA 92831, USA

${ }^{20}$ Columbia University, New York, NY 10027, USA

${ }^{21}$ Christopher Newport University, Newport News, VA 23606, USA

${ }^{22}$ Syracuse University, Syracuse, NY 13244, USA

${ }^{23}$ Stanford University, Stanford, CA 94305, USA

${ }^{24}$ Leibniz Universität Hannover, D-30167 Hannover, Germany

${ }^{25} \mathrm{OzGrav}$, University of Adelaide, Adelaide, South Australia 5005, Australia

${ }^{26}$ RESCEU, University of Tokyo, Tokyo, 113-0033, Japan.

${ }^{27}$ University of Birmingham, Birmingham B15 2TT, UK

${ }^{28}$ Cardiff University, Cardiff CF24 3AA, UK 
${ }^{29}$ The University of Mississippi, University, MS 38677, USA

${ }^{30}$ The Pennsylvania State University, University Park, PA 16802, USA

${ }^{31}$ Inter-University Centre for Astronomy and Astrophysics, Pune 411007, India

${ }^{32}$ University of Portsmouth, Portsmouth, PO1 3FX, UK

${ }^{33} \mathrm{OzGrav}$, University of Melbourne, Parkville, Victoria 3010, Australia

${ }^{34}$ OzGrav, School of Physics \& Astronomy, Monash University, Clayton 3800, Victoria, Australia

${ }^{35}$ The University of Texas Rio Grande Valley, Brownsville, TX 78520, USA

${ }^{36}$ University of Washington, Seattle, WA 98195, USA

${ }^{37}$ Universität Hamburg, D-22761 Hamburg, Germany

${ }^{38}$ Concordia University Wisconsin, 2800 N Lake Shore Dr, Mequon, WI 53097, USA

${ }^{39}$ Kenyon College, Gambier, OH 43022, USA

\begin{abstract}
The sensitivity of the Advanced LIGO detectors to gravitational waves can be affected by environmental disturbances external to the detectors themselves. Since the transition from the former initial LIGO phase, many improvements have been made to the equipment and techniques used to investigate these environmental effects. These methods have aided in tracking down and mitigating noise sources throughout the first three observing runs of the advanced detector era, keeping the ambient contribution of environmental noise below the background noise levels of the detectors. In this paper we describe the methods used and how they have led to the mitigation of noise sources, the role that environmental monitoring has played in the validation of gravitational wave events, and plans for future observing runs.
\end{abstract}

\title{
1. Introduction
}

Between 2010 and 2015, the two LIGO detectors at Hanford, WA (LIGO Hanford Observatory, or LHO) and Livingston, LA (LIGO Livingston Observatory, or LLO) underwent a period of extensive upgrades to transition from the Initial LIGO stage to the Advanced LIGO (aLIGO) configuration [1], significantly improving their sensitivity to gravitational waves [2]. The aLIGO detectors began their first observing run (O1) on September 12, 2015, and made the first detection of gravitational waves from a binary black hole (BBH) merger on September 14, 2015 [3], followed by two more BBH detections before the end of the run on January 16, 2016 [4]. The second observing run (O2) began on November 30, 2016 after a period of detector upgrades and ended on August 25, 2017. During O2, in addition to several more BBH detections, LIGO observed the first binary neutron star (BNS) merger on August 17, 2017 [5]. The third observing run (O3), which spanned April 1, 2019 to March 27, 2020, came after another round of major improvements in the performance of the detectors [6] and the full inclusion of the Virgo detector in the GW network. The first half of the run, ending on October 1, 2020, LIGO and Virgo observed a total of 39 GW events [7].

Environmental disturbances can significantly impact the data quality of the LIGO detectors. A gravitational wave (GW) is detected by measuring the differential arm length (DARM) of an interferometer (and converting it to a GW strain), so coupling between the external environment and the interferometer readout can reduce a detector's 
sensitivity to gravitational waves and potentially produce transient non-astrophysical signals in the detector. The environment can influence the detector through physical contact (via vibrations or temperature fluctuations), electromagnetic waves, static electric and magnetic fields, and possibly high-energy radiation. These effects are monitored with the physical environmental monitoring (PEM) system of sensors [8].

Studying environmental noise serves two purposes. The first is the validation of GW events. Environmental disturbances at amplitudes large enough to influence the LIGO data occur frequently around each detector and can potentially be correlated between different detectors, i.e. stemming from a common source as opposed to stemming from chance coincidence. Such correlated noise is not accounted for in the estimation of falsealarm probabilities, which is done by time-shifting background data from each LIGO detector to produce long stretches of coincident background. Environmental noise is particularly important in searches for un-modeled sources of gravitational waves, as these look for excess power without the use of waveform templates. Thus it is important to have a quantitative solution for identifying and evaluating the impact of environmental transients when they occur near candidate events.

The second purpose is to improve the sensitivity of the detector by reducing contamination from environmental noise. We track down troublesome noise sources and coupling mechanisms so that we can either remove the noise sources themselves, isolate them from the detector, or modify the detector to reduce coupling.

Effler et al. (2015) [8] described the methodology for studying environmental coupling and presented results from the sixth and final science run $(\mathrm{S} 6)$ prior to the transition to aLIGO. The methodology has since been improved and expanded, and sensitivity to environmental effects has changed with the upgrades to the detector. This paper describes these changes and presents cases where noise sources have been identified and mitigated between S6 and the end of O3. We also summarize how GW events are vetted using quantitative results from injections. This paper focuses on noise investigations at the LIGO detectors; a similar discussion for the Virgo detectors is provided in Fiori et al. (2020) [9].

There are many techniques for characterizing detector noise beyond those described here $[10,11,12,13,14]$. These include the use of tools for detecting excess power transients in the strain data [15], categorizing transients using machine learning to better distinguish them from astrophysical signals [16, 17], searching for correlated noise between auxiliary sensors and the strain data [18], and many more. Although these also play a role in achieving the goals above, this paper discusses more direct, focused techniques for studying, quantifying, and mitigating environmental effects.

This paper is organized as follows. In section 2 we summarize the changes made to the LIGO detectors and the PEM system since S6. In section 3 we present a method for quantifying environmental coupling based on data from noise injections. In section 4 we describe developments in the techniques for performing environmental noise injections. In section 5 we show results of recent studies and provide examples of how environmental influences have been mitigated. In section 6 we describe the process of vetting GW event 


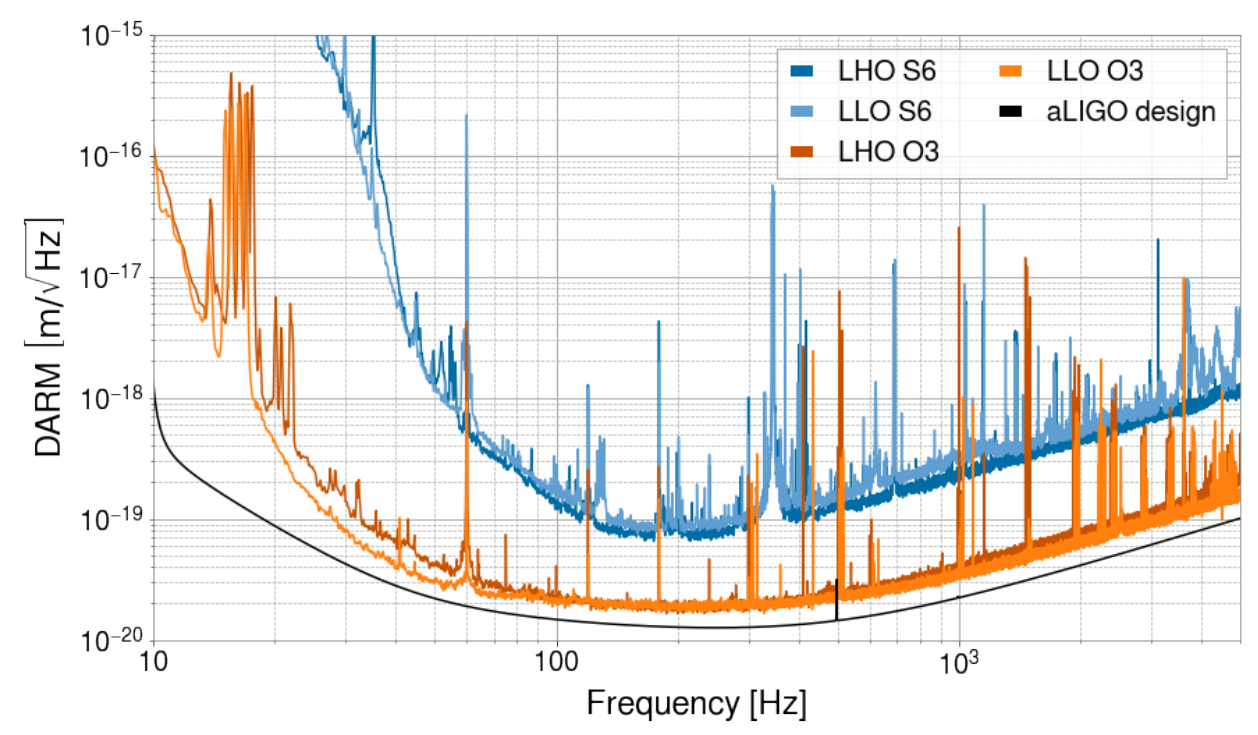

Figure 1. Amplitude spectral densities of the differential arm length displacement (DARM) at the end of S6 (Feb 272010 04:27:47 UTC) and during O3 (Mar 202020 00:00:00 UTC).

candidates with examples from real events. We conclude with a discussion of future work in section 7 .

\section{2. aLIGO Upgrades}

\subsection{Detector Upgrades}

When fully commissioned aLIGO is designed to provide an order of magnitude improvement to sensitivity in its most sensitive band [1], as well as more than an order of magnitude improvement at lower frequencies due to seismic isolation upgrades. Figure 1 compares the DARM noise spectra of LHO and LLO at the end of S6 to that at the end of O3. Significant progress has been made in approaching the design sensitivity of aLIGO, and further improvements are foreseen for the fourth observing run (O4), expected to begin in 2022. Here we highlight a few of the major upgrades that were directly relevant to reducing the coupling of ambient environmental noise.

The core interferometer optics (including the test mass mirrors and beam splitter) are suspended in active multi-stage suspension systems, which in turn are on active seismic isolation tables $[19,20]$. This provided a substantial improvement to sensitivity below $100 \mathrm{~Hz}$ over the initial LIGO configuration. The suspension and isolation tables also provide useful sensors for the motion of these optics.

Auxiliary sensors used in the control system of the interferometer were moved from in-air optical tables to in-vacuum, seismically isolated tables. This reduced acoustic coupling but did not eliminate it. Although the main laser system (PSL, or pre-stabilized laser) could not be moved into vacuum, an acoustically isolated room was built to house the laser, and a new optical table with improved isolation and damping of its resonances 
was installed [21].

To reduce magnetic coupling, magnets and certain magnetic materials are no longer present on or near the test masses themselves [22]. Instead, the aLIGO test masses are controlled either by magnets at the upper stages of the suspension system or by an electrostatic drive. To further reduce the ambient acoustic noise from electronics fans near the detector, power supplies and most electronics were moved to separate rooms (called here electronics bays), some tens of meters away from the vacuum system which houses the interferometer.

\subsection{Environmental Monitoring Upgrades}

Understanding environmental influences on the detectors requires comprehensive monitoring of its physical surroundings. This is done through the PEM system of auxiliary sensors, which consists of accelerometers for high-frequency vibrations (tens to thousands of $\mathrm{Hz}$ ), seismometers for low-frequency vibrations (up to tens of $\mathrm{Hz}$ ), microphones, magnetometers, voltage monitors that measure the voltage of electric power supplied to the detector sites, radio-frequency (RF) receivers, a cosmicray detector for high-energy particles, and wind, temperature and humidity sensors. Detailed information on PEM sensors, including example background spectra and calibration data, can be found on the PEM website, PEM.LIGO.org [23]. The site also provides links to long-term summaries of ground tilt, seismic motion, and wind (on the Environmental Studies pages).

In order to monitor environmental signals that could influence the interferometer, we use PEM sensors that are demonstrated to be much more sensitive to these signals than the interferometer is. Sensor locations are chosen with the goal of maximizing coverage of potential coupling sites. Ideally, if an environmental signal were to reach a coupling site, nearby sensors should be able to observe the signal at an amplitude equal to the amplitude at the coupling site. In practice, we place sensors where we expect the coupling to be strongest, and we may place new sensors during the run to improve monitoring of important coupling sites.

By focusing on the fundamental interactions that can affect the detector, the PEM system allows us to monitor potential effects from a large variety of environmental events. For example, wind can couple through vibrations in the ground and air, so its effects are monitored by seismometers, accelerometers, and microphones. Lightning could couple by magnetic fields, power mains disturbances, and electromagnetic waves at radio frequencies that we demodulate into the detection band, so lightning strikes are monitored with magnetometers, mains monitors, and RF receivers. The PEM sensors provide coverage of signals in the detection band of the interferometer $(20-2000 \mathrm{~Hz})$, although we also monitor beyond these frequencies when there are coupling mechanisms that convert low- or high-frequency signals up or down into the detection band or when the interferometer performance can be affected by frequencies outside of the detection band. 


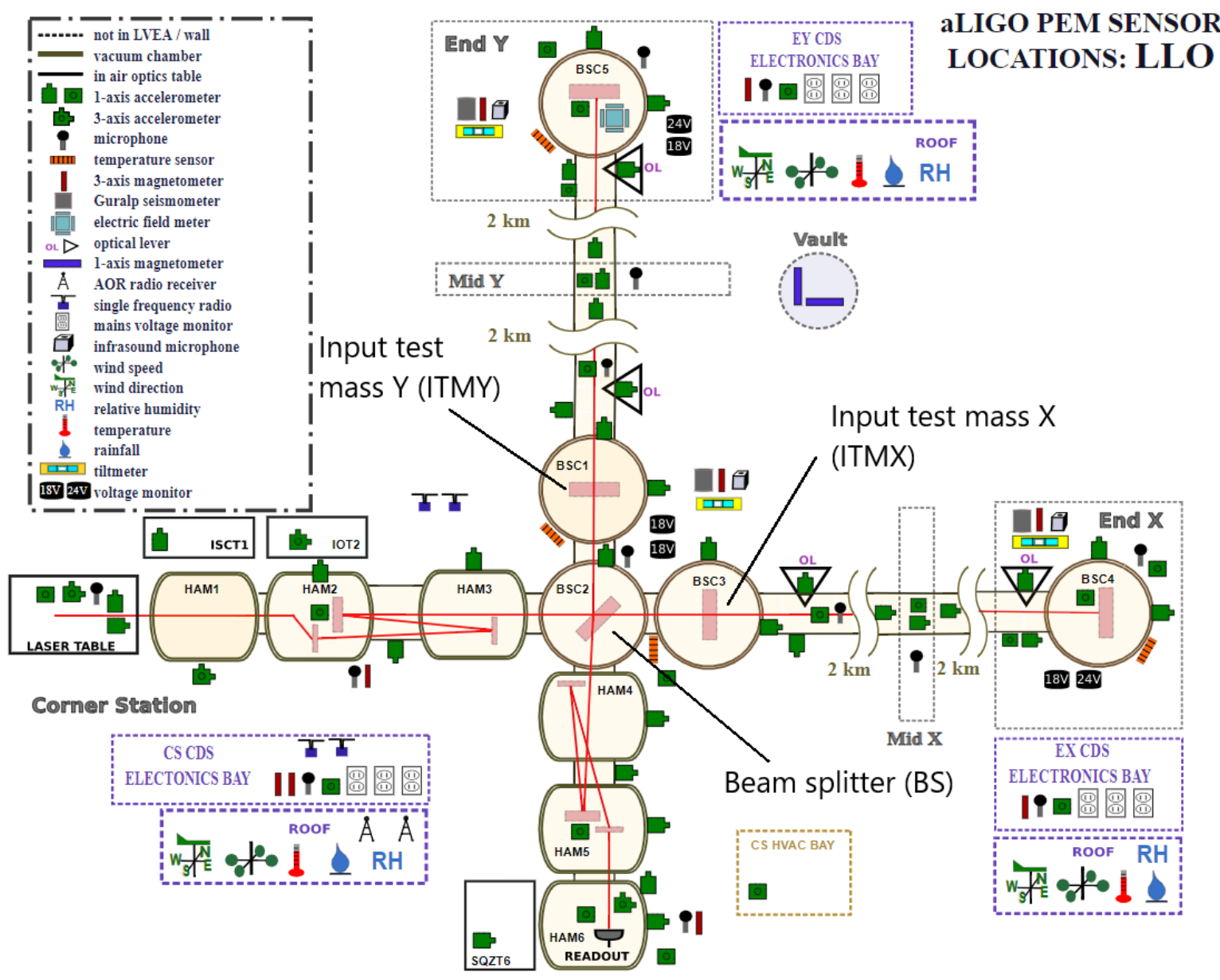

Figure 2. The Physical Environmental Monitoring system layout at the LIGO Livingston detector during $\mathrm{O} 3$, as seen on the PEM public website [23]. The path of the main interferometer laser is shown as a red line; core optics, such as the test masses, are represented by rectangles inside the vacuum chambers. The most major changes during aLIGO have been made to the accelerometer locations and the addition of new magnetometers, e.g. in the electronics bays.

The state of the PEM system at LLO during O3 is shown in Figure 2. A similar map for LHO is available on the PEM website [23]. Since the transition to aLIGO, many changes [24] have been implemented to expand the general coverage of the PEM system, provide additional monitoring near known high-coupling areas, and adapt to the detector upgrades described in 2.1. Many changes involved the addition of accelerometers or relocation of existing ones:

- Vacuum chambers: In iLIGO, most accelerometers were mounted on the seismic isolation system. These locations became redundant with the introduction of vibrational sensors as part of the new active isolation systems, so the accelerometers are now mounted on the chamber walls where they can detect motions that could modulate laser light scattered off of the chamber walls.

- Beam tubes: Accelerometers at select sites along the 4-km beam tubes monitor 
vibrations that affect the modulation of reflected light inside. Coverage now includes the mid-stations, which is especially important at LHO where significant coupling has been measured, likely because they contain the smallest aperture between vertex and end stations.

- Electronics bays: Floor accelerometers were added to detect vibrational coupling to the electronics boards (e.g. through resistance variations in poor solder joints) and to monitor the rooms as seismic sources.

- Vacuum enclosure areas: Floor accelerometers were added near the vacuum chambers in order to expand coverage and aid in localizing sources of vibrations through propagation delays and amplitude differences at locations that do not have the resonance structure of the vacuum envelope.

- Pre-stabilized laser table: Coverage of the main laser table was expanded. This area has continued to be a major source of vibrational coupling.

Many sensors were also upgraded to newer models in order to improve their performance. Table 1 summarizes the current sensor models and specifications.

Magnetometer coverage was also expanded, particularly with the addition of magnetometers on the electronics racks (located in the electronics bays) which were important noise sources and coupling sites during initial LIGO. Relatively large magnetic fields are generated by the equipment in the racks and these fields can couple to components, cables and, connectors in the racks. Additionally, magnetometers in electronics racks have been useful for identifying sources of narrow spectral peaks even when the coupling was not through magnetic fields. Cyclical processes producing line artifacts in the DARM spectrum can be tracked down by detecting currents associated with those processes. In a sense, we monitor multiple electronic systems at once, using fluxgate magnetometers in the electronics racks (Bartington-03 series [25]). These are sensitive enough to detect periodic currents with amplitudes as low as $5 \times 10^{-5} \mathrm{~A}$ at 1 $\mathrm{m}$ from long wires or traces [26].

Additionally, the non-rigid tripods for fluxgate magnetometers were replaced with rigid ones. Non-rigid tripods lead to increased cross-talk between floor vibrations and the magnetometer signal, as the magnetometer vibrates relative to the Earth's magnetic field at the tripod resonance frequency. This created a peak in the magnetometer spectrum a factor of three above background, which was eliminated by switching to rigid tripods [27].

In addition to the fluxgate magnetometers that monitor local magnetic fields, extremely low frequency induction coil magnetometers (LEMI-120 [28]) were added to the PEM system in order to monitor magnetic noise from Schumann resonances. These are global electromagnetic resonances in the cavity formed by the Earth's surface and the conductive ionosphere. Lightning strikes around the world excite this resonant cavity, producing picoTesla-scale magnetic fields that can cause correlated noise in the LIGO detectors [29, 30, 31]. Two LEMI magnetometers are positioned at each site, far enough from the detector so that they are not sensitive to the same local magnetic fields 
Table 1. Specifications for important PEM sensor types. The operating frequency range is the range in which the sensor calibration is flat; we often use them over a broader range. Noise floor numbers are reported in the operating band of each sensor (seismometer at $1 \mathrm{~Hz}$ ).

\begin{tabular}{|c|c|c|c|c|}
\hline Type & Sensor & Operating freq. & Sampling freq. & Noise floor \\
\hline seismometer & Guralp $^{\circledR}$ CMG-3T $[34,35]$ & $0.1-20 \mathrm{~Hz}$ & $256 \mathrm{~Hz}$ & $<1 \mathrm{~nm} / \mathrm{s} / \sqrt{\mathrm{Hz}}$ \\
\hline accelerometer & Wilcoxon $\left.{ }^{\circledR}\right) 731-207[36]$ & $1-900 \mathrm{~Hz}$ & $4096 \mathrm{~Hz}$ & $0.5 \mu \mathrm{m} / \mathrm{s}^{2} / \sqrt{\mathrm{Hz}}$ \\
\hline microphone & Brüel\&Kjær ${ }^{\circledR} 4130$ [37] & $10-900 \mathrm{~Hz}$ & $16384 \mathrm{~Hz}$ & $<30 \mu \mathrm{Pa} / \sqrt{\mathrm{Hz}}$ \\
\hline microphone & Brüel\&Kjær ${ }^{\circledR} 4188[38,39]$ & 8-12500 Hz & $16384 \mathrm{~Hz}$ & $<5 \mu \mathrm{Pa} / \sqrt{\mathrm{Hz}}$ \\
\hline magnetometer & Bartington ${ }^{\circledR}$ 03CES100 [25] & $0-900 \mathrm{~Hz}$ & $8192 \mathrm{~Hz}$ & $<6 \mathrm{pT} / \sqrt{\mathrm{Hz}}$ \\
\hline magnetometer & LEMI-120 ${ }^{\circledR}[28]$ & $0.0001-1000 \mathrm{~Hz}$ & $4096 \mathrm{~Hz}$ & $<0.1 \mathrm{pT} / \sqrt{\mathrm{Hz}}$ \\
\hline radio station & $\mathrm{AOR}^{(\mathbb{R}}$ AR5000A [40] & $24.5 \mathrm{MHz}$ & $16384 \mathrm{~Hz}$ & - \\
\hline
\end{tabular}

observed by the fluxgate magnetometers. They are placed at a location between the corner station and end stations, 100-200 $\mathrm{m}$ from the beam tube, one aligned with the $x$-axis and one with the $y$-axis of the interferometer.

An electric field meter was installed in an end test mass chamber at each observatory $[32,33,6]$. These can detect electric fields generated inside of the chambers as well as fields from outside the chamber that make it in through glass viewports on the chambers.

\section{Coupling Functions}

To determine the degree to which the detector is affected by environmental influences during operation, we inject basic environmental disturbances that produce a response in DARM. We make acoustic injections with speakers and monitor them with the system accelerometers and microphones; seismic injections with shakers, monitoring them with the accelerometers and seismometers; magnetic injections with wire coils monitored with the magnetometers. The injection methodology is described in more detail in Section 4. To motivate the injection techniques we first discuss the means of quantifying the coupling.

Suppose there exists only one coupling site, a sensor is placed at the location of the coupling site, and a noise injection is performed that produces a signal in the sensor and some response in DARM. A coupling function can be computed based on the actuation measured by the witness sensor and the response measured in DARM [41, 42]. We compare the amplitude spectral densities (ASDs) of DARM and the witness sensor during the time of the injection (injection time) to their ASDs during a time when both are at observation-mode noise levels (background time). The coupling function at some frequency $f$ is given by

$$
\mathrm{CF}(f)=\sqrt{\frac{\left[Y_{\mathrm{inj}}(f)\right]^{2}-\left[Y_{\mathrm{bkg}}(f)\right]^{2}}{\left[X_{\mathrm{inj}}(f)\right]^{2}-\left[X_{\mathrm{bkg}}(f)\right]^{2}}}
$$

where $X_{\mathrm{bkg}}(f)$ and $X_{\mathrm{inj}}(f)$ are the ASDs of the witness sensor at background and 
injection times, respectively, and $Y_{\mathrm{bkg}}(f)$ and $Y_{\mathrm{inj}}(f)$ are the ASDs of DARM at background and injection times. We use coupling factor to refer to the value of a coupling function at a single frequency bin.

A sensor's coupling function can be used to compute the contribution of noise in the sensor to DARM. For example, when validating GW events, we multiply the coupling function by the amplitude of any environmental transient observed by the sensor to predict the corresponding amplitude in DARM. Additionally, multiplying the coupling function by the sensor's ambient background level yields the ambient contribution of noise at the sensor to the DARM spectrum: $Y(f)=\mathrm{CF}(f) X(f)$.

Suppose now we expand the scenario such that there are multiple coupling sites, and a sensor is placed at the location of each site. We can model the response in DARM to each injection as a linear combination of the sensor signals and their sensor-specific coupling functions. To solve for the coupling functions, we can perform multiple injections instead of just one, resulting in a system of $n$ equations with $m$ unknown coupling functions, where $n$ and $m$ are the numbers of injections and sensors, respectively:

$$
Y_{i}(f)=\sum_{j=1}^{m} \mathrm{CF}_{j}(f) X_{i j}(f) .
$$

Here $Y_{i}(f)$ and $X_{i j}(f)$ are the amplitudes of DARM during injection $i$ and sensor $j$ during injection $i$ respectively, and $\mathrm{CF}_{j}(f)$ is the coupling function of sensor $j$. One could solve (2) to determine the coupling functions of all sensors.

We have assumed thus far that the witness sensors are placed at the locations of the coupling mechanisms, but such perfect placement is not realistically feasible given that there are an unknown number of coupling sites at unknown locations. A sensor, even if it is near a coupling site, only measures the injection amplitude at its own location, not at the coupling location. Therefore, when using real-world sensors, (1) is only an estimate of the true coupling, and (2) is not an exact model of all the coupling mechanisms. Nevertheless, as explained above, we distribute sensors to maximize coverage of coupling sites and find that this has been sufficient for producing reliable coupling functions for all sensors, as discussed further in Section 3.1.

One hurdle remains in attempting to solve (2). In practice, typically $n<m$ due to logistical constraints on the number of injections one could perform during a realistic time window, which makes the system of equations underdetermined. The problem can be simplified by instead approximating $\mathrm{CF}_{j}(f)$ for each sensor independently of other sensors. Given a sensor $j$, we can re-purpose (1) (replacing $X$ with $X_{i j}$ and $Y$ with $\left.Y_{i}\right)$ to compute a single-injection "coupling function" $\mathcal{C F}_{i j}(f)$ for each injection, then combine those to produce an approximation to $\mathrm{CF}_{j}(f)$. The closer an injection is to a sensor, the more accurate the computed $\mathcal{C F}_{i j}(f)$ would be to $\mathrm{CF}_{j}(f)$, since the DARM response would be dominated by coupling near sensor $j$. Since it is impractical to produce an injection at each sensor, the approach we have adopted for combining the $\mathcal{C F}_{i j}(f)$ is to construct a composite coupling function whose value at each frequency bin 
is the coupling factor corresponding to the nearest injection, determined by the highest sensor amplitude (using the assumption that injection amplitudes are equivalent). That is, for a frequency $f_{k}$ and a set of injections $\mathcal{I}$, we measure the sensor amplitudes $\left\{X_{i j}\left(f_{k}\right) \mid i \in \mathcal{I}\right\}$, compute the single-injection coupling functions $\left\{\mathcal{C F}_{i j}\left(f_{k}\right) \mid i \in \mathcal{I}\right\}$, and compute the composite coupling function as

$$
\widetilde{\mathrm{CF}}_{j}\left(f_{k}\right):=\mathcal{C F}_{l j}\left(f_{k}\right) \text { where } l=\underset{i \in \mathcal{I}}{\operatorname{argmax}}\left(X_{i j}\left(f_{k}\right)\right) \text {. }
$$

If the distribution of injection locations provides sufficient coverage of sensor locations, then $\widetilde{\mathrm{CF}}_{j}(f) \approx \mathrm{CF}_{j}(f)$. We discuss shortcomings of this assumption in Section 3.1.

Computing the single-injection coupling functions $\mathcal{C F}_{i j}(f)$ (example shown in Figure 3) requires a significant difference between the injection and background signals in the sensor and in DARM. To distinguish between measurements and upper limits, thresholds are chosen for the sensor and DARM in the form of a ratio between the injection ASD and background ASD. For each frequency bin, if an injection produces a large enough signal to exceed both the sensor threshold and DARM threshold, then a coupling factor can be measured via Eq. 1. If the injection exceeds the sensor threshold but not the DARM threshold, then we instead compute an upper limit by omitting the DARM background term. These thresholds are typically chosen to be a factor of two in DARM and a factor of a few in the sensor, based on the typical level of fluctuations observed in the spectra.

The composite coupling function computed via (3) is used for comparing coupling between different sensor locations and producing estimates of DARM amplitudes, e.g. as part of event validation (see Section 6). Therefore we refer to a sensor's composite coupling function simply as its coupling function from here on. Figure 4 provides an example of an estimated ambient for an accelerometer on the HAM6 vacuum chamber (which houses the interferometer output optics). The PEM website provides coupling functions for all accelerometers, microphones, and magnetometers produced from the most recent campaign of injections [23].

\subsection{Uncertainties and Limitations}

We characterize coupling using the coupling function defined in (1) instead of a transfer function because we do not assume perfect coherence. Low coherence can arise either due to non-linearity in the coupling or due to the spacing between the sensor and coupling site.

To measure coupling, we inject signals large enough to produce a response in DARM, but the maximum amplitude of injections is limited by the sensitive range of the environmental sensors (saturation produces an overestimate of coupling). This effectively limits how far below the DARM background we can probe for coupling or establish upper limits. 

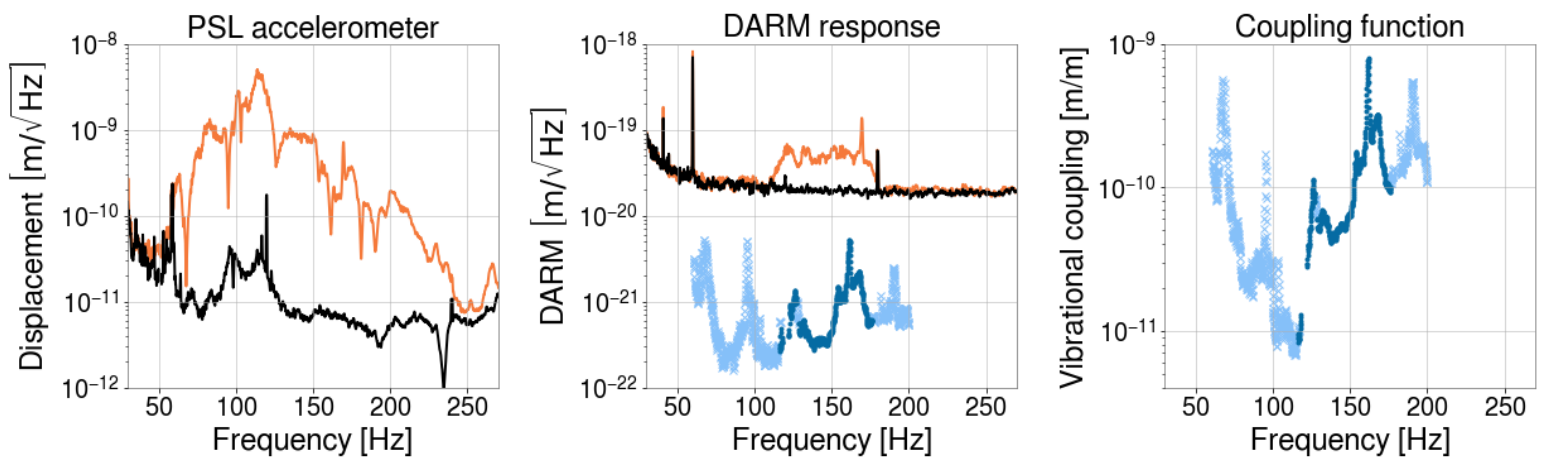

Figure 3. Vibrational coupling excited by a broadband $(60-200 \mathrm{~Hz})$ acoustic injection near the output arm of the interferometer. The left plot shows the displacement of an accelerometer in the PSL room during background time (black) and injection time (orange). The middle plot shows the interferometer readout during background time (black) and injection time (orange). Estimated ambient levels for the accelerometer are also shown as dark blue dots, with upper limits shown as light blue crosses; they are produced from the single-injection coupling function in the right plot. A vibrational single-injection coupling function represents meters of differential test mass displacement per meter of sensor displacement, hence the units of $\mathrm{m} / \mathrm{m}$.

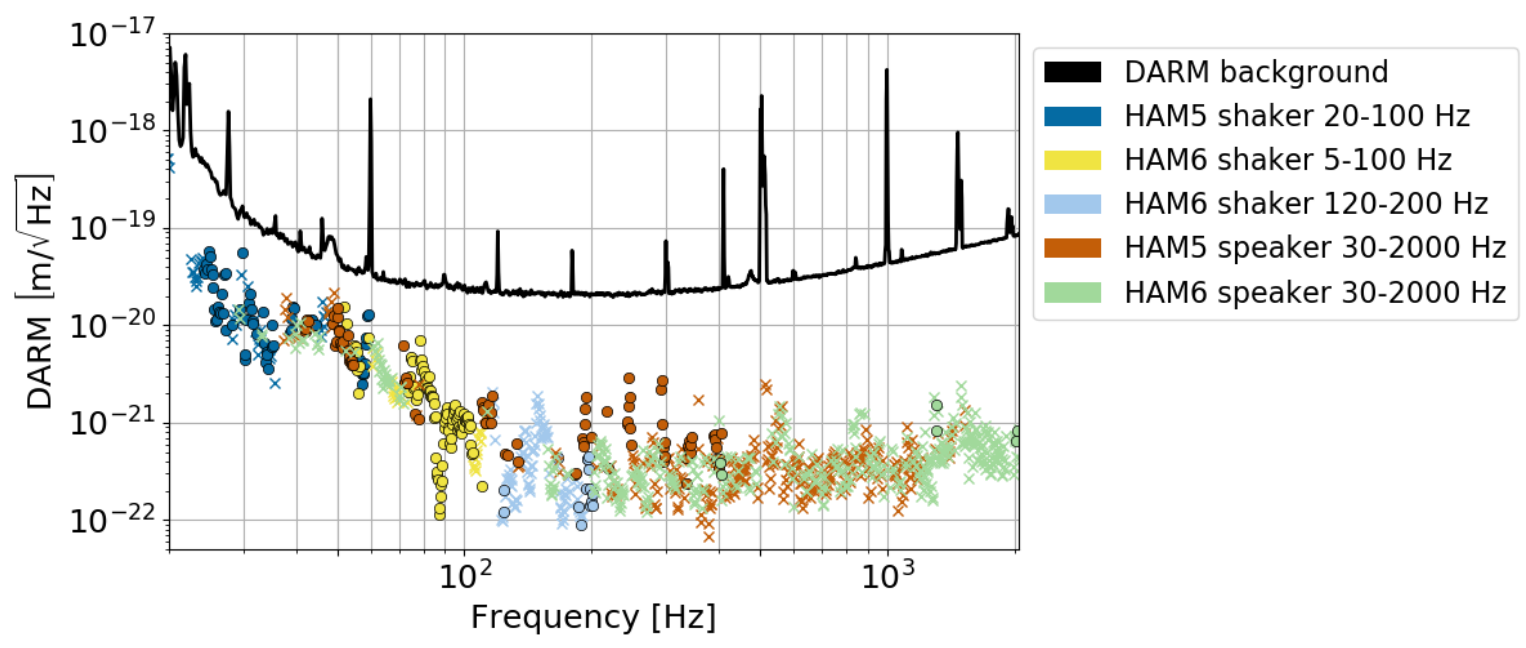

Figure 4. Ambient noise level for the LHO HAM6 Y-axis accelerometer estimated from a composite coupling function, using acoustic and seismic injections near the output arm. For simplicity only five injections were used to produce this example, however in practice the number of injections performed near a sensor can be many times higher.

Equation (1) relies on two assumptions about the coupling mechanism. First, the coupling is assumed to linear, e.g. doubling the amplitude of the injection would double the amplitude of the response in DARM. We check this by repeating injections with different amplitudes. Second, the coupling function ignores any up- or down-conversion of the signal between the sensor and DARM. This non-linear coupling can be very significant for scattering noise and bilinear coupling but is not accounted for in the estimates of linear coupling. One way we detect non-linear coupling is by sweeping 
single frequency injections over time and searching for off-frequency response in DARM spectrograms. Frequency changes from non-linear coupling can be an issue in broadband injections where up- or down-converted noise in DARM appears in the injection band, resulting in artificially higher estimates of linear coupling. We split broadband injections into smaller frequency bands to avoid this effect when necessary. One approach for quantifying non-linear coupling is presented in Washimi et al. (2020) [43].

As mentioned above, the use of (2) relies on the assumption that the environment is monitored at the coupling site. The density of sensors is not great enough for this to be strictly true, especially if the source of the environmental signal is closer to the coupling site than the sensor is. The finite spacing of sensors leads to imperfect coupling functions but, for environmental signals that are generated at a distance greater than the typical sensor spacing of a few meters (the external signals that are the focus of PEM), the uncertainty can be estimated based on the differences between injections made at different locations. We choose a sensor near a known coupling site and find the variance between single-injection coupling functions measured for that sensor. Figure 5 shows single-injection coupling functions for an accelerometer measured from shaker injections produced from three locations. Since the injection locations are close enough to the accelerometer, we can assume that the variance is entirely due to the distance between the sensor and the coupling site. Variations in injection location result in a multiplicative scaling of the single-injection coupling functions, so we quantify the variance by computing the geometric standard deviation of coupling factors in each bin. Averaged across all bins, the geometric standard deviation between injection locations is 1.4 , i.e. coupling functions measured from vibrational injections, as well as vibrational noise projections to DARM, vary by a factor of 1.4. A similar study combining geometric standard deviations for various magnetometers at both observatories shows that magnetic coupling measurements vary by a factor of 1.7 [44].

In the case of acoustic injections, the uncertainty in a coupling function can be exacerbated when nodes and anti-nodes in the acoustic signal coincide with the location of a sensor. This results in peaks and troughs in the sensor spectrum at frequencies that have a node or anti-node at the sensor location, respectively. These artifacts can impact any sensor, but are more noticeable in microphone spectra than accelerometer spectra, possibly because the stiffness of the vacuum enclosure results in effectively averaging over a larger area; in microphones, the peak-to-trough ratio is typically a factor of a few. The peaks and troughs are present in the sensor but not in DARM, because the sensor monitors a single point whereas the coupling to DARM is spread across a large enough area for the effects of nodes and anti-nodes to average out. Consequently, this effect imprints troughs and peaks onto the coupling function. The artifacts can be smoothed out of the spectra by computing a moving average over $X_{\text {inj }}(f)$. The peakto-peak distances are typically a few $\mathrm{Hz}$, so we smooth the spectra enough to remove features up to a few $\mathrm{Hz}$ across. This is acceptable in broadband acoustic injections which are designed to not produce any other spectral features at this scale.

Although the injections used to measure coupling functions are designed to best 


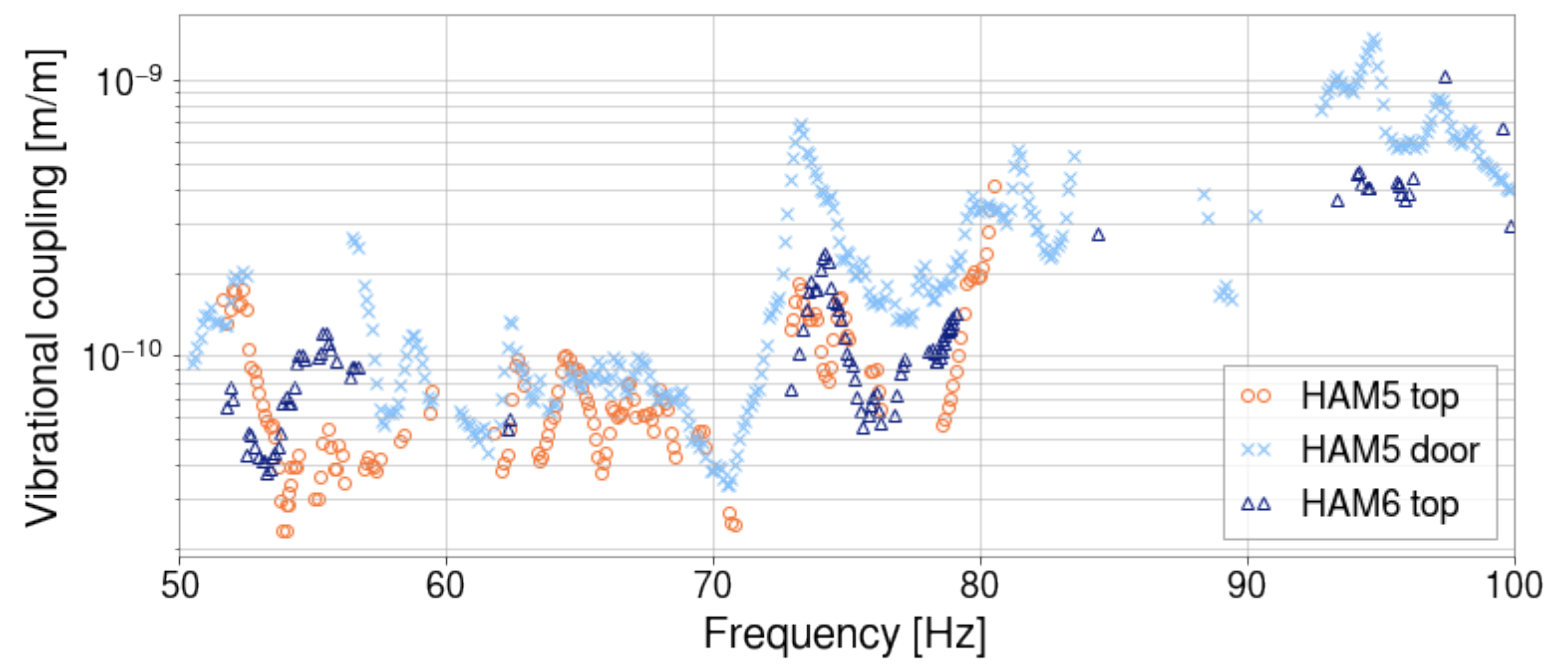

Figure 5. Single-injection coupling functions for the HAM5 Y-axis accelerometer from shaking injections made from three different locations (on top of HAM5, on top of HAM6, and on the HAM5 chamber door) show the typical spread in coupling that results from varying the injection location. Multiple injections at different frequency bands are shown for each source location. On average the coupling measured from different locations varies by a 1.4 .

replicate environmental noise, there are still differences and it is useful to test the coupling functions with different environmental events by comparing noise seen in DARM during such events to noise levels predicted by PEM sensors and their coupling functions. Thunderstorms are known to produce short-duration transients in DARM at tens of $\mathrm{Hz}$. At LLO, coupling functions for several accelerometers at the $\mathrm{Y}$ end station, where vibrational coupling was the highest, were capable of estimating the amplitude of multiple transients in DARM to within a factor two during a particularly loud thunderstorm [45]. Helicopter flyovers can produce narrow-band features in DARM up to tens of seconds long. Coupling functions of various sensors at both interferometers predicted the amplitudes of lines produced by multiple helicopter flyovers during O3 to within a factor of two in most cases [46]. Vibrational noise from rain and the building HVAC, which produce much longer-duration noise in DARM, have also been well estimated by coupling functions at LHO [47, 48].

\section{Injection Methods}

The basic methodology of environmental noise injections is described in [8]. Here we summarize the methods and describe improvements made to the hardware and techniques since then.

Injection locations are chosen to best mimic disturbances from outside the detector. To do so we choose them to be as far from the detector and environmental sensors as possible, but we are usually limited by the size of the detector sites themselves (some injections can be made from outside). We perform injections from as many locations as 
Table 2. Specifications for injection equipment.

\begin{tabular}{ll}
\hline Equipment & Injection type \\
\hline Custom enclosure with two 14-in. speakers & Acoustic \\
Various smaller speakers & Acoustic \\
APS 113 Electro-Seis ${ }^{\circledR}$ Long Stroke Shaker [49] & Vibrational \\
Piezosystem ${ }^{\circledR}[50]$ shaker with custom reaction mass & Vibrational \\
Brüel \& Kjær ${ }^{\circledR}[51]$ EM shaker with custom reaction mass & Vibrational \\
$1 \mathrm{~m}$ diameter copper coil $(100$ turns) & Magnetic \\
$3 \times 3 \mathrm{~m}$ and $5 \times 5 \mathrm{~m}$ coils $(80-100$ turns) & Magnetic \\
\hline
\end{tabular}
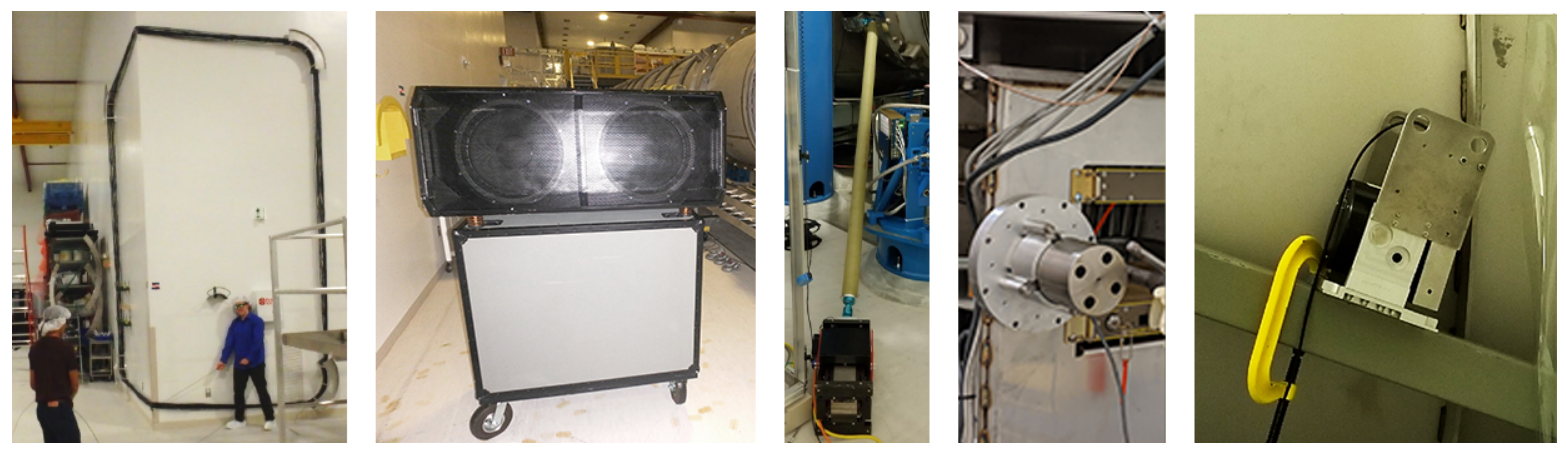

Figure 6. Injection equipment photos. From left to right: wall-mounted magnetic field injection coil; 14-in. speakers; APS 113 shaker connected to the door of a vacuum chamber by a rigid fiberglass rod; modified Piezosystem shaker clamped to an electronics rack; modified B\&K shaker clamped to a beam tube support.

time allows in order to maximize coverage of potential coupling sites. Increased time allocation towards environmental studies in recent years has allowed for a significant increase in the number of injection locations.

Table 2 summarizes the current equipment used and Figure 6 shows photos of some of the equipment. Seismic injections at low frequency (up to tens of $\mathrm{Hz}$ ) during initial LIGO were performed with small electromagnetic and piezoelectric shakers and a weighted cart. A large shaker [49] has been used since the beginning of noise studies for O3.

Two new injection techniques have been developed for localizing vibration coupling sites connected to the vacuum enclosure, such as locations on the vacuum enclosure that reflect scattered light. The techniques rely on the slow propagation speeds (hundreds of meters per second) of vibrations on the steel vacuum enclosure walls or, for acoustic injections, in air.

The first technique is narrow-band, and involves vibrating the vacuum enclosure at two slightly different frequencies, each injected from a shaker or a speaker at a different location (e.g. a shaker at one location injects a sine wave at frequency $f$ and a shaker at the other location injections at frequency $f+0.01 \mathrm{~Hz}$ ). The two injections are 


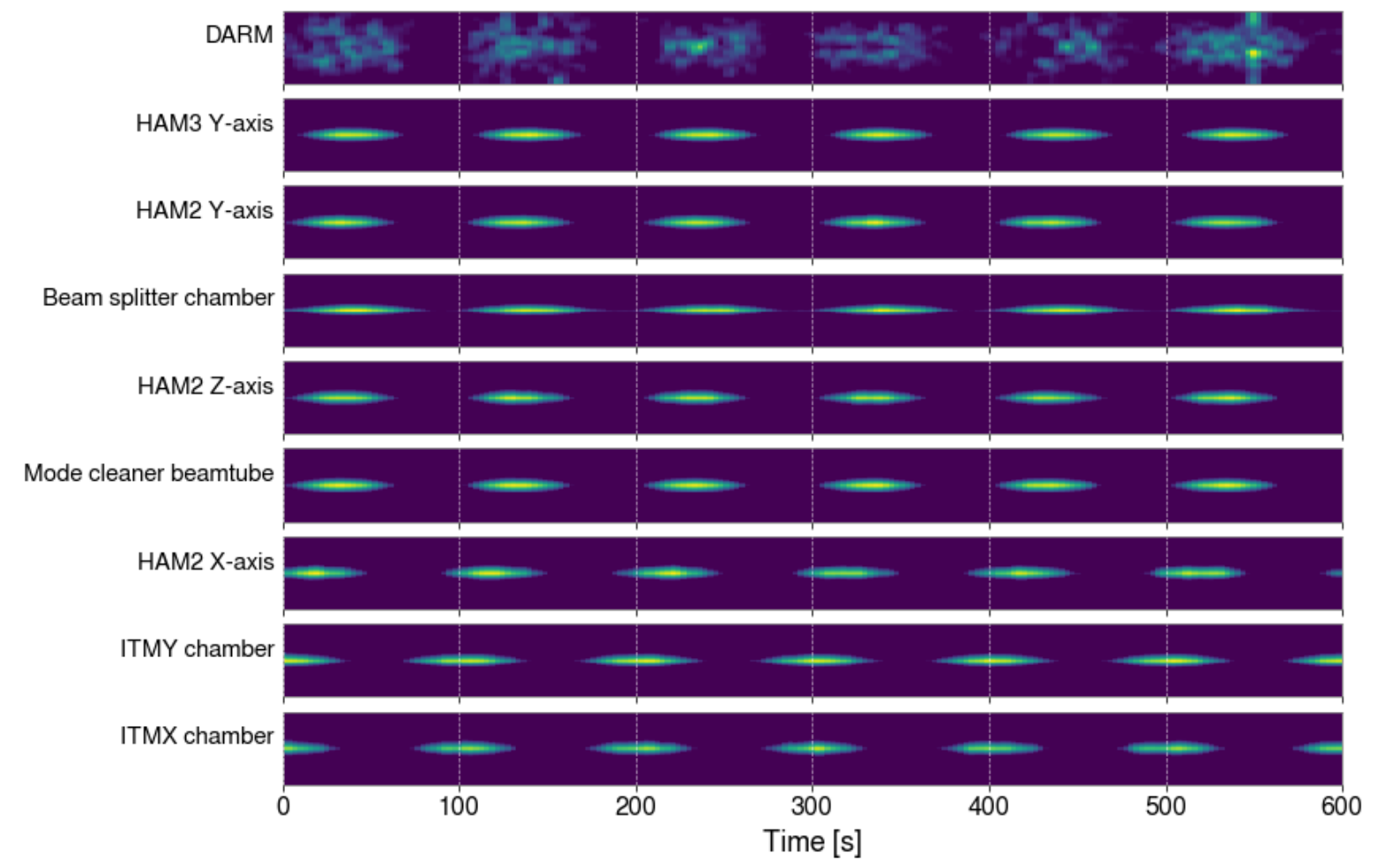

Figure 7. Example spectrograms showing a vibrational beat injection using two shakers to localize the coupling site responsible for a $48 \mathrm{~Hz}$ peak in the DARM spectrum. The shakers were injecting at 48 and $48.01 \mathrm{~Hz}$. The Y-axes of the spectrograms are centered along at $48 \mathrm{~Hz}$ and show the combined signal in each sensor modulating at the beat frequency $(0.01 \mathrm{~Hz})$. This set of spectrograms suggests that the accelerometers on the input test mass (ITM) chambers and the Y-axis HAM2 accelerometer are likely not close to the true coupling location, since the beat envelopes are the furthest offset from the beat envelope in the DARM response. Multiple other injections were made (not shown here) with varying shaker locations in order to rule out other sensors until the most likely candidate remaining was the HAM3 Y-axis accelerometer. Black glass was used to block scattered light at this location and the peak was eliminated for the second half of the O3 observation run.

adjusted in amplitude to produce strong beats in DARM. Because the injection locations are different, the relative phase of the two injected signals varies with location on the vacuum enclosure. As a result, the phase of the beat envelope varies with position, and different sites experience maximum chamber wall motion at different times. The sites with accelerometer signals that have the same beat envelope phase as DARM are candidates for the scattering sites on the vacuum enclosure walls (Figure 7). Other sensors that are not near the coupling site may also match the phase by chance, but these false positives can be rejected by varying the locations of the shakers.

The second injection technique, which is broad band, involves propagation delays in impulse injections. Impulse injections are performed by striking the vacuum enclosure directly with enough force to produce a transient in DARM and in nearby accelerometers. The vibrational impulse propagates through the structure of the vacuum 
enclosure, arriving at different accelerometers and coupling sites at different times. We can distinguish these arrival times because the propagation velocity is much slower than in solid material, and is only roughly $300 \mathrm{~m} / \mathrm{s}$ in our case. Using time series plots, the arrival time of the impulse in DARM is compared to the arrival time of the impulse in multiple accelerometers (Figure 8, left). The accelerometers that have the same arrival time as DARM are more likely to be near a coupling site than those that observe the impulse much earlier or later than DARM does. Again, varying the location of the injection eliminates sensors that match the DARM time-of-arrival by chance but are actually far from the coupling site. An additional consistency check is that the coupling of accelerometers near the coupling site will vary less between different impulse locations than that of accelerometers far from the coupling site. Finally, if the accelerometer is at the coupling site, the impulse in DARM will have a resonance structure that is similar to the resonance structure of the accelerometer signal, which can be judged from spectrograms (Figure 8, right).

These two techniques aided in the localization of a coupling site that was producing a $48 \mathrm{~Hz}$ peak in DARM throughout the first half of O3 [52]. The peak was present before the start of the run, and shaker and acoustic injections suggested the source was likely at the corner station. Impulse injections pointed to the highest coupling being near the vertex and input arm. Using the double-shaker beat injection method, with frequencies of 48 and $48.01 \mathrm{~Hz}$, it was found that the timing of the beat envelope in DARM best matched that of the HAM3 door, even after varying the shaker locations and using a temporary accelerometer to test other nearby locations. This led to the discovery that the $48 \mathrm{~Hz}$ peak was a result of scattered light at the HAM3 viewports, which was promptly eliminated by blocking it with black glass, removing the $48 \mathrm{~Hz}$ peak from the DARM spectrum for the remainder of the observing run.

Improvements have also been made to the magnetic field injection equipment. In order to generate fields strong enough to couple into DARM using the $1 \mathrm{~m}$ magnetic field coils made during initial LIGO [8], we must focus the power of the coil into narrow bands and combs instead of injecting broadband signals. This was sufficient in initial LIGO when strong magnetic coupling occurred primarily through permanent magnets. However, due to the removal of permanent magnets from the test masses, coupling from those sources has decreased and cables and connectors have become the dominant coupling sites above about $80 \mathrm{~Hz}$, introducing more structure to the coupling functions and requiring stronger injections.

To achieve high-amplitude broadband magnetic injections, seven wall-mounted coils, each one a $3 \mathrm{~m} \times 3 \mathrm{~m}$ or $5 \mathrm{~m}$ x $5 \mathrm{~m}$ square of 80-100 turns, are being installed at each site; three at the corner station and two at each end station. These coils are fixed in place and can be operated remotely, allowing for weekly injections to monitor variations in magnetic coupling caused by changes to electronics. Figure 9 compares the old and new magnetic injections. Some coils were installed and operated at the sites during O3; the project will be completed by the start of $\mathrm{O} 4$. 

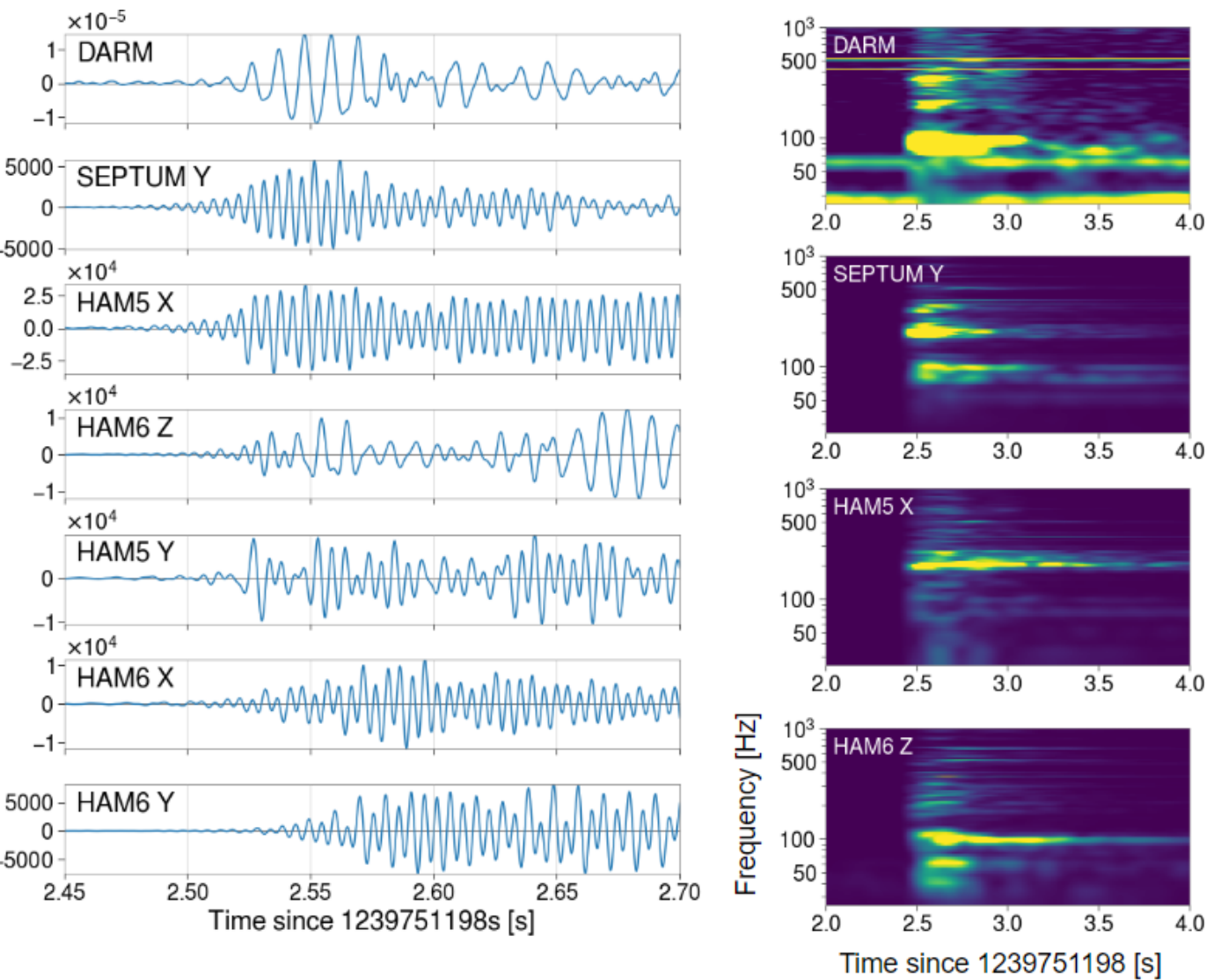

Figure 8. Left: Example time series of a single impulse injection signal in DARM and various output optics accelerometers. Multiple sensors observe an impulse timeof-arrival matching that of DARM, but repeating the injection from various other locations rules out sensors that do not match DARM consistently across multiple injections. In this case the septum (separating the HAM5 and HAM6 chambers) accelerometer signal matched the DARM signal most consistently (other injections not shown for brevity). Right: Spectrograms of the same impulse injection for DARM and the three sensors with the closest matching time-of-arrival to DARM. The similarity between the frequency structure of the septum accelerometer and that of DARM further supports the septum as a dominant coupling site in the output arm.

\section{Mitigation of Environmental Effects in aLIGO}

We use the methods discussed thus far to track down noise sources whose estimated ambient level in DARM is more than a tenth of the DARM background. Mitigation can be accomplished in three ways: by removing or modifying the source itself, by isolating the source or otherwise addressing propagation of the signal to the detector, or by reducing the coupling itself through some modification to the detector. Here we provide several examples of environmental effects that were mitigated based on results from noise investigations. 

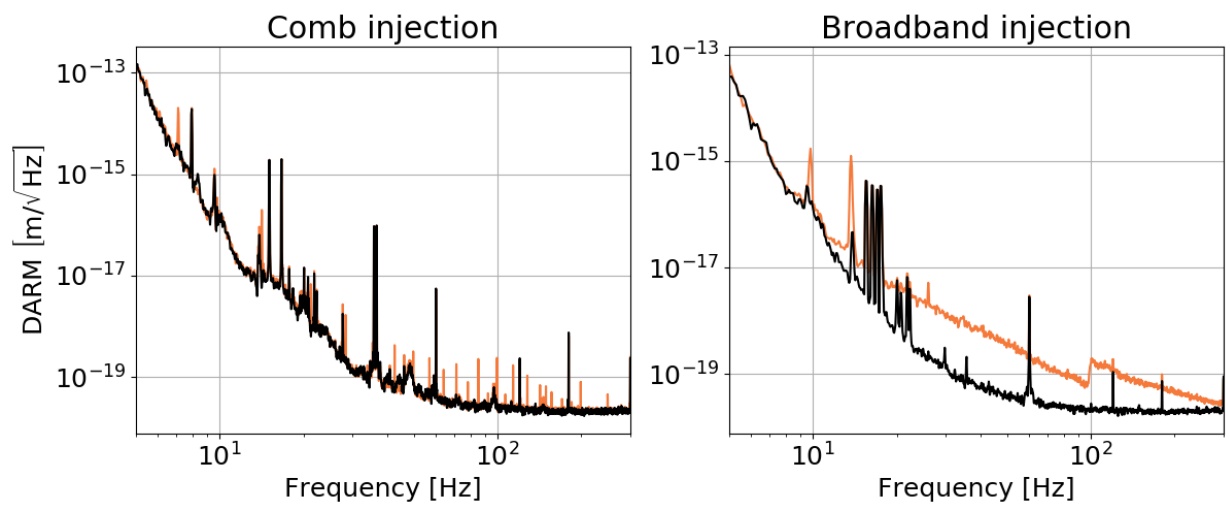

Figure 9. DARM response to old (left, comb) and new (right, broadband) magnetic injections. Black and orange lines show the DARM ASD before and during the injections, respectively. The comb injection curve is a composite of multiple comb injections, with fundamental frequencies of $7.1 \mathrm{~Hz}, 14.2 \mathrm{~Hz}$, and $49.7 \mathrm{~Hz}$, made at different times. The broadband injection spectrum is a composite of a 10-100 $\mathrm{Hz}$ injection and a 100-1000 Hz injection made at different times, hence the break at 100 Hz.

\subsection{Seismic and acoustic influences}

Figure 10 shows the ambient contribution of vibrational noise during O3, produced by combining the highest coupling factors among accelerometers and microphones measured from an injection campaign at the beginning of O3. At the end of O3, the vibration noise background at both observatories was dominated by input beam jitter above 100 $\mathrm{Hz}$ (discussed in Section 5.1.1). At LHO, the dominant coupling region below $100 \mathrm{~Hz}$ was the output arm. At LLO, the dominant coupling regions were the Y-end in the 40-60 $\mathrm{Hz}$ band and the output arm in the 60-100 $\mathrm{Hz}$ band.

5.1.1. Input beam jitter. Alignment fluctuations of the beam (beam jitter) entering the interferometer cause variation in the coupling of the fundamental mode into the arm cavities, producing amplitude noise. In addition, the varying beam position relative to defects in the test masses causes a variation in the balance of light between the two arms, further contributing to the noise [6]. The dominant source of alignment fluctuations was turbulence in the laser cooling system, causing vibration of mirrors and other optics on the table and in the laser, producing peaks in DARM at mechanical resonances of the optics and their mounts. A second mechanism may be variation in beam diameter associated with the turbulent cooling. In addition to vibrations from the cooling system, transient vibrations, such as those made by large vehicles or heavy footsteps in the control room, produced transients in DARM by temporarily increasing alignment fluctuations.

Mitigation has included removing turbulence-producing connectors, sharp turns in the coolant lines, and abrupt diameter changes within the cooling system; and reducing the flow of coolant [53]. In addition, injections were used to identify the optic mounts 

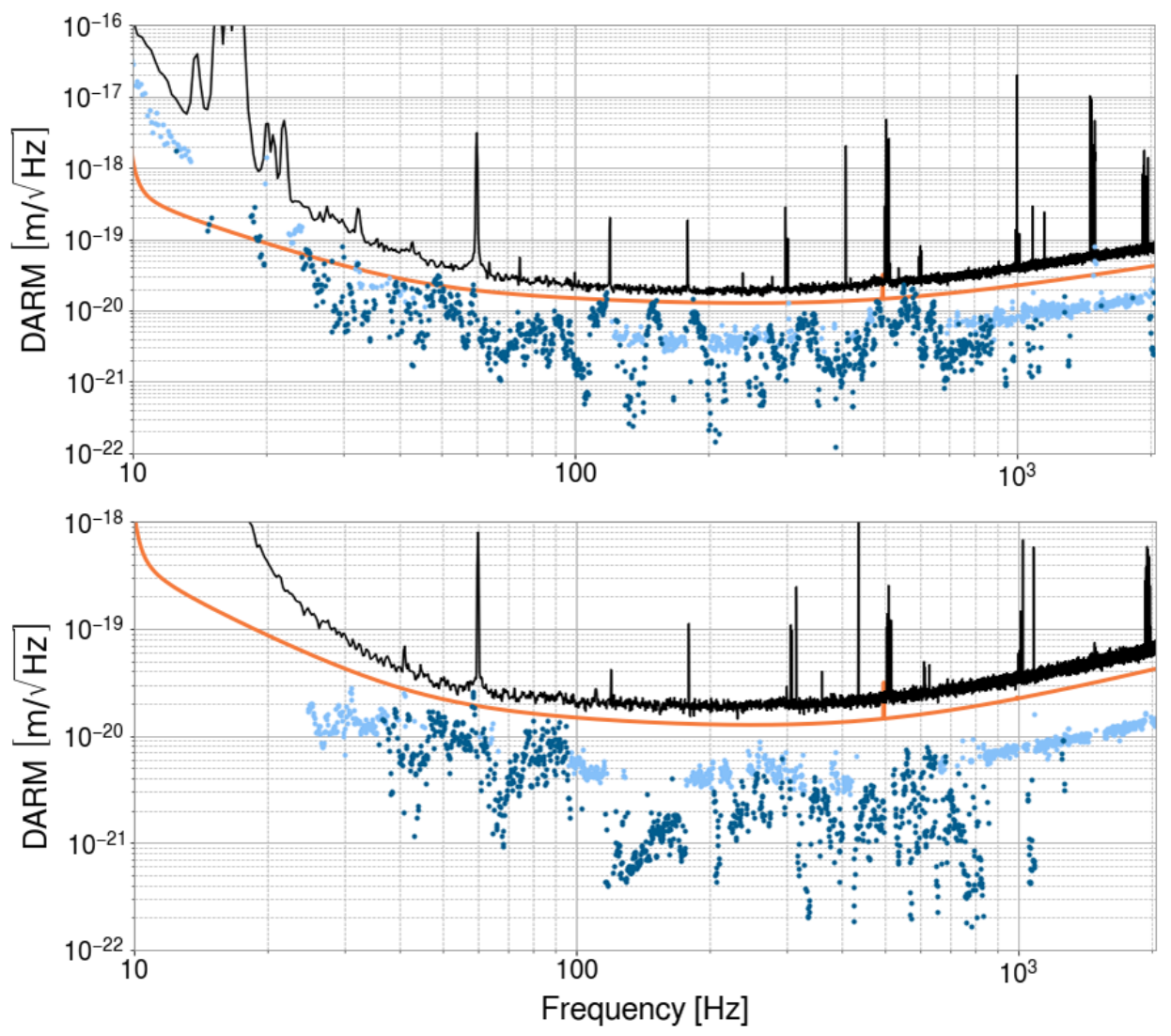

Figure 10. Ambient vibrational noise at LHO (top) and LLO (bottom), shown in dark blue (measurements) and light blue (upper limits). The values are produced by selecting the highest-amplitude composite coupling function at each bin across all sensors at each observatory. The black and orange lines show the DARM background and the aLIGO design sensitivity, respectively.

that produced the largest peaks in DARM, and mass-and-viton dampers were added to the mounts. This resulted in motion reductions by factors of a few [54]. Finally, the resonances of optic mounts on the periscope that raises the beam from the laser table level to the interferometer level, were tuned by adding small masses to the optic mounts, so that their resonances would not overlap in frequency with periscope resonances that would increase the motion of the mounts [55].

5.1.2. Vibrational coupling at resonances of the vibration isolation system. One of the most troubling environmental couplings early in aLIGO was vibration coupling at 100 $\mathrm{Hz}$ and above through the seismic isolation system. Not only were ambient vibration levels producing noise within a factor of two of the DARM background around 1000 $\mathrm{Hz}$, but the coupling was highly non-linear (see non-linearity discussion in Uncertainties and Limitations), and it was the only vibrational coupling observed that could produce noise in the detection frequency band around $100 \mathrm{~Hz}$ from a source near $1000 \mathrm{~Hz}$. One could imagine a rising frequency signal (chirp) from the startup of a motor with squealing bearings, for example, that would have been able to produce a chirp in DARM 
in the $100 \mathrm{~Hz}$ band. This problem required special vetting of the first GW detections since, normally, the vetting procedure only assumes linear coupling (discussed further in Section 6).

The coupling was due to little or no isolation in certain frequency bands associated with mechanical resonances of the isolation system. The active system vibrationally isolating the in-vacuum optical tables works mainly below $20 \mathrm{~Hz}$. For higher frequencies, there are one (HAM chambers) or two (BSC chambers) passive layers associated with the suspension of the optical tables. But, at the many resonances (violin modes) of the multiple wire-like flexures that suspend the tables, there was little isolation, allowing vibration at these frequencies to couple to DARM. This lack of isolation produced linear vibration coupling at multiple optical tables and, at the dark port table, nonlinear coupling due to an intermodulation of vibration and a strong length dither used in controlling the length of the output mode cleaner (OMC). The coupling was mitigated by attaching Viton ${ }^{\mathrm{TM}}[56]$ to the suspension flexures at tables with coupling to DARM. To further reduce non-linear coupling, the amplitude of the OMC length dither was reduced as far as possible [57].

5.1.3. Coupling of wind through ground tilting in the $0.1 \mathrm{~Hz}$ band. Vibrations from wind affect the interferometer directly in the 10-100 Hz band. At lower frequencies, particularly in the band around $0.1 \mathrm{~Hz}$, pressure fluctuations associated with wind can affect performance and duty cycle by tilting the ground. Performance can be affected by direct tilt of optical table supports or by tilt of ground motion sensors used in the active isolation system, producing inaccurate signals from sensors that do not distinguish between tilt and acceleration. Even far from the buildings, we found that the ground tilts in wind (about 1e-8 radians/sqrt(Hz) at $0.1 \mathrm{~Hz}$ in wind reaching $15 \mathrm{~m} / \mathrm{s}$ at LHO), to a degree that is consistent with spatially varying wind speeds and Bernoulli forces. But the tilting in the buildings was a factor of several times larger, and found to be greatest near the building walls. The pressure fluctuations on the walls are thought to tilt the wall supports which, in turn, tilt the ground at their base. The coherence length of floor tilt measured at Hanford was a couple of meters, indicating that the cement slab does not tilt as a unit. Instead, the tilting is local and mainly within meters of the base of columns that support the walls, consistent with an elastic dimpling of the ground around the support [58, 59].

The localized nature of the dominant tilt has led to the simple mitigation technique of moving ground sensors as far from the walls as possible. While certain sensors could be moved, the large vacuum chambers near the wall could not, and for future installations, we have recommended that the chambers be placed at least $10 \mathrm{~m}$ from the base of wall supports.

In order to further mitigate the effects of wind-induced tilt, tilt meters with improved sensitivity were designed and deployed [60, 61]. The first versions were produced to correct the artifacts that tilts produce in seismometers, but a table-top tilt meter is also being developed in order to mitigate the effects of the tilt of the optical 
tables in the chambers.

Wind fences have been used to reduce wind in agricultural and recreational settings, and modeling suggested that wind fences may be useful for reducing the effects of wind pressure on the building walls. For this reason a wind fence was constructed at Hanford, and is currently being evaluated [62]. One remaining question is how effective a wind fence is in the troubling frequency band around $0.1 \mathrm{~Hz}$ where the length scale is $100 \mathrm{~m}$ for $10 \mathrm{~m} / \mathrm{s}$ wind.

5.1.4. Vibration modulation of scattered light paths. A major source of detector noise and reduced sensitivity to GWs is the scattering of light from the beam spot on a test mass or other optic to surfaces that are moving relative to the optic, like vacuum chamber walls. A very small fraction of the light reaching the moving surface is reflected to the originating or another beam spot, where it scatters back into the main interferometer beam. As the distance to the moving surface changes, the phase of the returning light changes relative to the main beam, producing fluctuations in the amplitude of the beam, that, at 1 part in $10^{20}$ can be on the scale of those produced by gravitational waves. In addition to this sensitivity to recombined scattered light, the scattering noise is problematic because of non-linear coupling when the path length modulation becomes comparable to the wavelength of the light, producing noise at harmonics of modulation frequencies [63].

The subtlety of scattered light noise is illustrated by the mechanism that was behind a mysterious glitch in DARM that turned out to be produced by ravens [64, 12]. The Rube Goldberg-like mechanism began in the desert sun at LHO, where ravens pecked at ice accumulations on a cryopump vent tube just outside of an end station building. The vibrations from pecking were transmitted through the vent tubes to the cryopump inside the building. The cryopump was attached to the beam tube, and the vibrations were transmitted through the beam tube to a calibration structure located inside of the vacuum, which vibrated slightly with each peck. The structure was angled so as not to retro-reflect light scattered from the test mass, about $10 \mathrm{~m}$ away. However, polishing grooves on the surface reflected a small fraction of the light back to the test mass, where a small fraction recombined with the main beam. The interference between the light in the main beam and the tiny amount of light reflected from the grooves varied with the motion of the calibration structure produced by each peck. The varying interference caused fluctuations in the light of the main beam, similar to the fluctuations produced by gravitational waves. After the discovery of this coupling mechanism, the calibration structure was baffled to reduce the light scattered back into the interferometer, eliminating the raven glitches and other similar vibrational signals.

Scattered light baffles can themselves be problematic - for example, vibrations in the 5-30 Hz band, such as from nearby truck traffic, produced transient noise and limited detector sensitivity in early aLIGO. Investigations using vibration injections and laser vibrometry showed that the coupling was due to light reflecting from imperfect light baffles. The ground motion was amplified by the resonances of the baffles (quality factors 
of several hundred), increasing the velocity by several hundred times, and producing scattering noise that reached hundreds of $\mathrm{Hz}$ for $10 \mathrm{~Hz}$ excitations [65]. The problem was solved by damping the baffle resonances with Viton ${ }^{\mathrm{TM}}$ in order to reduce their velocity [66]. Eventually, the most reflective parts of the baffles were also removed. Near the end of O3, a second type of undamped baffle was identified as a noise source [67] [68] and damped [69] (Figure 11).

Scattering noise can be mitigated by reducing the amplitude of the scattered light or by reducing the velocity of the reflector, or both. Reducing the amplitude by a factor of two yields a factor of two reduction in noise; reducing the velocity instead reduces the maximum frequency of the noise by the same factor. In the latter case, most of the noise above the newly lowered cutoff frequency is removed which can lead to a greater overall reduction of the noise than just a factor of two [63].

An important driver of scattering noise for long interferometers is ground motion at the ocean-wave driven microseismic peak frequency, in the 0.1-0.3 Hz region, which has produced noise in DARM that reached nearly $100 \mathrm{~Hz}$, several hundred times higher in frequency. Because the ground moves differently at the ends of the 4-km-long cavities, the control systems that minimizes relative motion of the test masses at opposite ends of the cavity can lead to micron-scale relative motion between the end test mass (ETM) and other objects in its vicinity that are not moved with the test mass and cavity. A major source of transients during the first three observing runs, especially when microseismic noise was high, was light reflected from the gold electrostatic actuator traces on the reaction mass behind the test mass. The variation in the optical path length was amplified by multiple reflections between the traces and the back of the reflective surface of the test mass [70]. The problem was solved by driving the reaction mass to minimize relative motion between it and the test mass [63].

Diagnostic photographs can be used to identify a common type of scattering path that involves light that is scattered from the beam spot on an optic to a moving reflective surface and back to the beam spot, where it scatters back into the main beam (Figure 12). Problematic reflective surfaces often depend strongly on precise angles and surface finish, and they can be difficult to identify in design drawings. To find potential reflective surfaces, during incursions such as optic installation, we place a small camera (with the camera flash very near its aperture) as close to the face of an optic as possible, and look for bright reflections of the flash in photographs taken from the optic's point of view (12). Most metal surfaces that would directly reflect infrared light scattered from the face of the optic also directly reflect camera flashes from the face of the optic. Since reflections are common and it is difficult to fix each one, we have begun work on a system to roughly rank the noise potential of reflective surfaces in the photographs, using the estimated coupling of scattered light to the GW signal at the particular optic, the solid angle of the reflecting surface and its distance from the optic, the approximate motion of the surface, and the estimated angular dependence of the scattering from the optic along with the angle to the reflector [71]. 

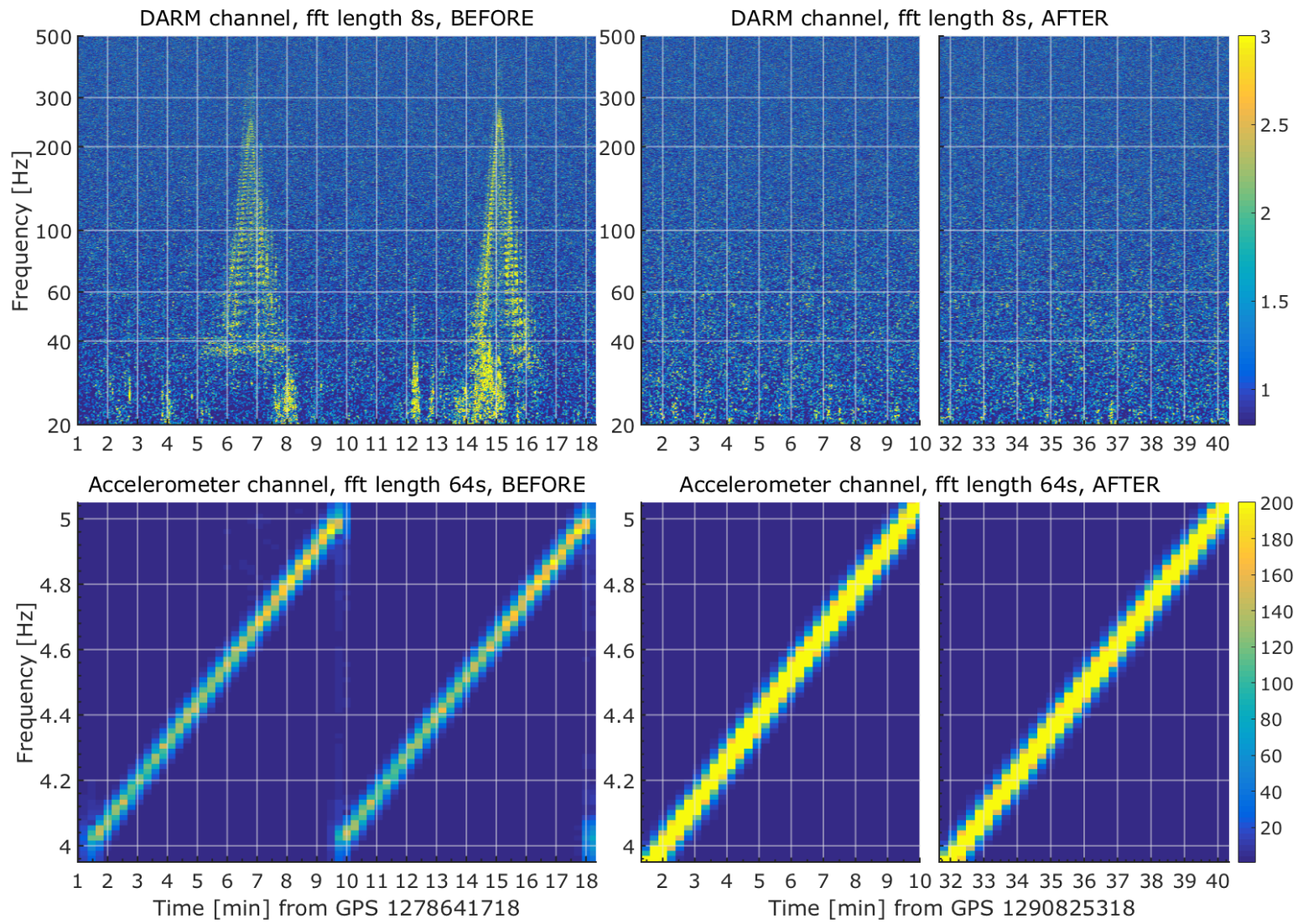

Figure 11. Spectrograms of the DARM channel (top) and an accelerometer channel (bottom) showing consecutive vibrational sweep injections that excite noise in DARM as they cross $4.62 \mathrm{~Hz}$ before mitigation. The "AFTER" sweep is composed of two graphs such that it shows only the relevant range of the sweep to be compared with "BEFORE". The spectrograms are normalized by median, but the spectra are not very different between the two tests such that they are directly comparable. The $\mathrm{Q}$ was determined to be around 450 and so it is easy to get to high velocities by exciting this resonance - identified on a baffle at the end station. This resonance was damped in between the two measurement sets, such that the same sweep with even higher amplitude no longer makes any noise in DARM.

\subsection{Magnetic influences}

Magnetic injections early in aLIGO suggested that coupling to permanent magnets in the suspension system could prevent LIGO from reaching design sensitivity in the 10-20 $\mathrm{Hz}$ regions [72]. While the test mass actuator is electrostatic and not magnetic (as in initial LIGO), a number of permanent magnets were used in the suspensions, including for actuation in the first three of the four levels of the isolation chain and for eddy current damping. The greatest number of permanent magnets were in the eddy current damping arrays and these were removed. Nevertheless, ambient fields are still predicted to produce noise at greater than one-tenth of the design sensitivity in the $10-20 \mathrm{~Hz}$ band (Figure 13), and may need to be further addressed as we reach design sensitivity in the 


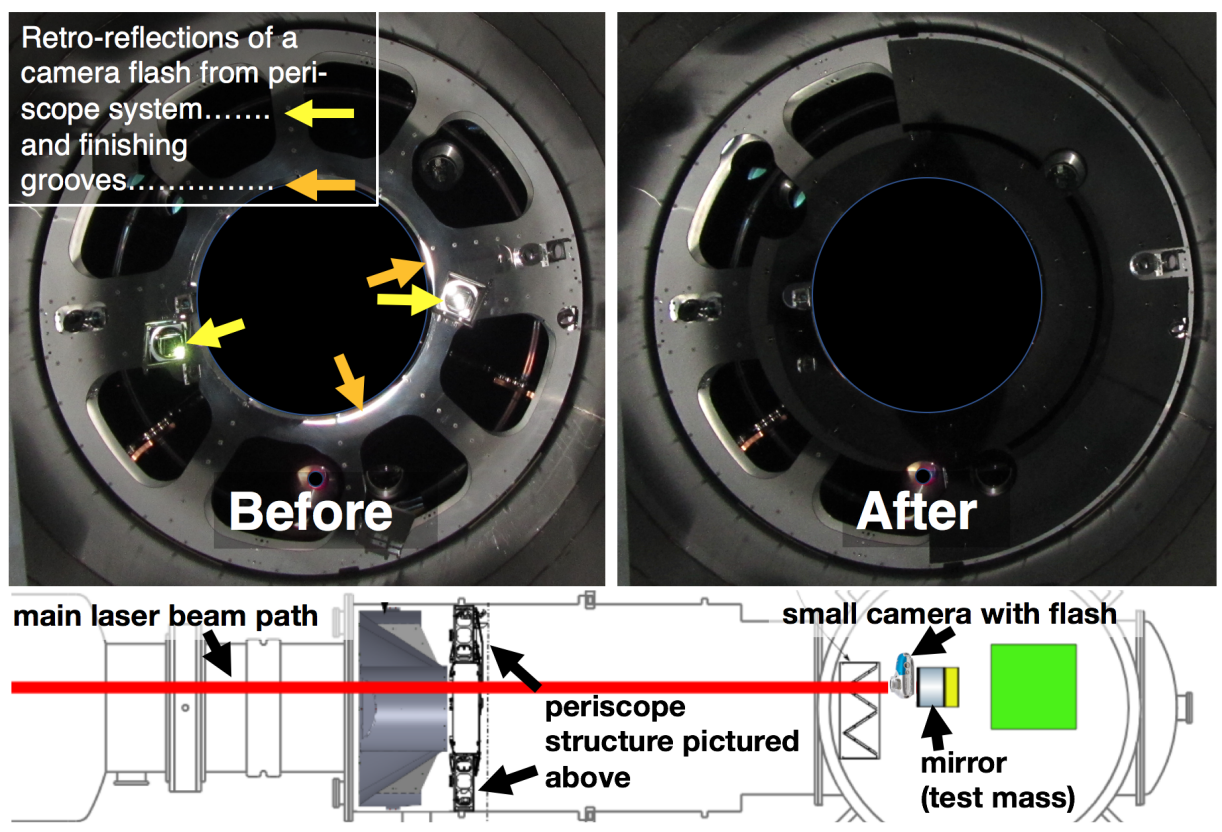

Figure 12. Diagnostic photographs taken from the point of view of a test mass beam spot, before and after scattering noise mitigation. The image on the left shows retroreflections of light from the camera flash, which follows a similar path as light scattered from the interferometer beam by imperfections in the test mass. The photograph was used to identify potential sources of scattering noise. The structure is a calibration periscope in the beam tube. The photograph on the right was taken after removal of the mirrors and after the subsequent installation of baffling, both of which reduced the scattering noise in the GW channel. The central black disk was added to the image to avoid confusion from light reflected by a gate valve that is withdrawn during operation.

\section{$10 \mathrm{~Hz}$ region.}

At higher frequencies, generally above about $30 \mathrm{~Hz}$, the dominant magnetic coupling appears to be through induction of currents in cables and at connectors, mainly to actuator cabling and other cabling in the control system. Mitigation of coupling to cables and connectors has required a continuing program of monitoring coupling since cables are often disconnected and reconnected during runs as electronics are replaced for problems or upgrades. This program consists of making weekly, broadband magnetic field injections using the large wall-mounted coils described in 4 . The injections have shown that peaks can appear or disappear, as well as shift in frequency, on a weekly basis.

As explained in Section 2.2, magnetometers can be used to identify sources of persistent spectral artifacts in DARM, even when the coupling mechanism is not necessarily through magnetic fields. Many examples of lines and combs mitigated throughout $\mathrm{O} 1$ and $\mathrm{O} 2$ through magnetometer studies are provided in Covas et al. (2018) [26].

While ambient fields do not normally limit the sensitivity of the interferometers for most astrophysical sources, the stochastic GW searches reach higher strain sensitivities 

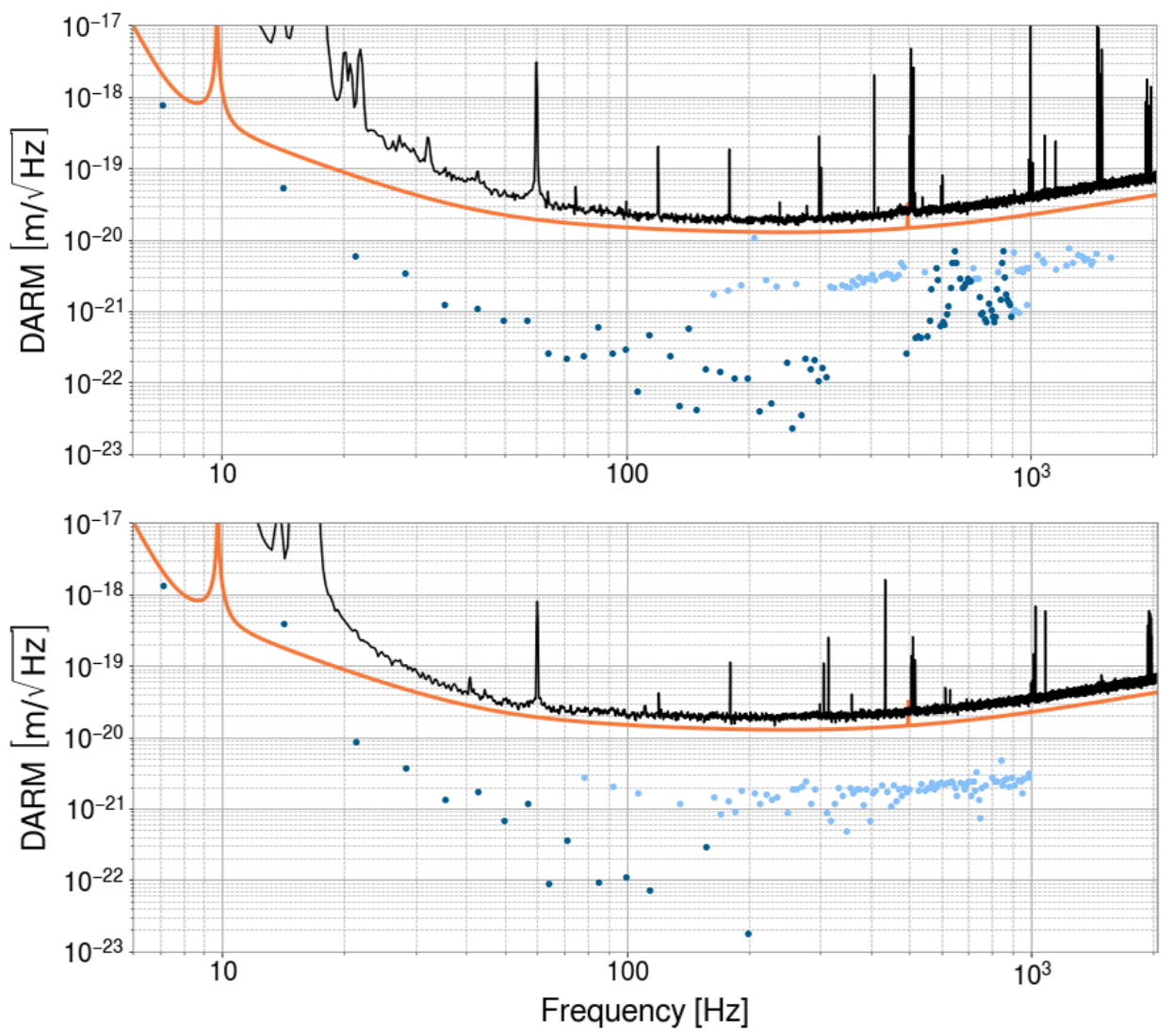

Figure 13. Ambient magnetic noise at LHO (top) and LLO (bottom), shown in dark blue (measurements) and light blue (upper limits). The values are produced by selecting the highest-amplitude composite coupling function at each bin across all sensors at each observatory. Only values at multiples of $7.1 \mathrm{~Hz}$ are used because most of the injections performed were $7.1 \mathrm{~Hz}$ comb injections (off-comb bins had too few injections to produce reliable composite coupling functions). The black and orange lines show the DARM background and the aLIGO design sensitivity, respectively.

by integrating data over multi-month periods and searching for correlations between sites. Magnetic fields that are correlated between sites could limit this sensitivity or even lead to misinterpretations of the GW background. Geomagnetic phenomena, such as the Schumann resonances discussed in Section 2.2, could produce such correlations $[29,30,31]$. In order to monitor such correlated fields, we have installed sensitive magnetometers far from the much greater uncorrelated magnetic fields in the buildings.

\subsection{Other influences}

5.3.1. Dust and particulates. In initial LIGO, the photodiodes at the interferometer output were external to the vacuum system and glitches were produced in DARM by dust passing through the beams at the photodiodes, generally where the beams were smallest in diameter. For aLIGO, the photodiodes were placed in vacuum, but the main laser was not. The room containing the main laser is a clean room and we have not found dust to be a problem. However, excess vibration of the beam tube and vacuum 
chambers can cause oxidized metal particulate from the inside of the vacuum system to drop through the beams and produce glitches in DARM. An absence of a correlation between glitches in DARM and ground motion suggests that this problem is minimal, though it has shown up strongly when the beam tubes were being cleaned and when the vacuum chambers are struck [73]. For the beam tube, we estimated that particulate glitches were unlikely for accelerations less than $1 \mathrm{~m} / \mathrm{s}^{2}$ [74], which is still a concern because it could be reached in stick-slip events associated with thermal expansion of the beam tube.

5.3.2. Radio-frequency fields. Injections have shown that out-of-band RF fields (above $10 \mathrm{kHz}$ ) have no influence on the interferometer until they reach amplitudes that are orders of magnitude above most of the background from external sources [75]. The strongest RF coupling was found to be at the 9 and $45 \mathrm{MHz}$ modulation frequencies. We monitor these frequencies and we scan for signals between $9 \mathrm{kHz}$ and $1 \mathrm{GHz}$.

Unlike externally generated RF fields, RF signals generated by the detector have been observed to produce noise in the gravitational wave channel, glitches called RF whistles [11] and other features. For example, during the first observing run, a narrowband feature intermittently appeared in the LHO DARM spectrum around $650 \mathrm{~Hz}$, resulting in spurious GW event candidates near that frequency [76]. The feature was found to be correlated with a similar feature observed by the RF receiver. The receiver actually witnessed the RF noise during a particular step of the interferometer's lock acquisition process, the transition to DC readout. This eventually led to discovering that the origin of the noise was the frequency of a voltage-controlled oscillator (VCO) used in lock acquisition beating with some other RF source in the inteferometer. Changing the frequency setting of the VCO eliminated the $650 \mathrm{~Hz}$ line from DARM [77].

5.3.3. HVAC and other temperature control systems. A dominant source of vibration in the $1-10 \mathrm{~Hz}$ band has been air and cooling water flow associated with the large central HVAC systems in the corner and end stations. Turbulent eddies in the air plenum downstream of the HVAC turbines were shown to produce vibrations that affected the interferometer. The large eddies producing pressure fluctuations in the few $\mathrm{Hz}$ region were broken up by installing screens in the outlets of the turbines, reducing ground vibration in the band around $5 \mathrm{~Hz}$.

Water chillers and pumps used to chill the HVAC system are generally isolated on springs, but turbulence in the pipes connecting the chillers to the air handling system was observed to increase ground motion in the $5-15 \mathrm{~Hz}$ region. The turbulence was partially mitigated by reducing the chilled water flow using variable frequency drives to slow the water pumps. Isolation of the pipes from contact with the ground and larger diameter pipes could reduce this problem in future installations.

Temperature fluctuations, while generally in the $\mathrm{mHz}$ band, can affect interferometer performance by, for example, changing the length of the blade springs at the top of the pendulum suspensions, which lowers or raises the entire test mass 
suspension, resulting in rubbing. Building temperature control often employs sensors mounted on the walls. When these walls are external, the temperature in the region of the vacuum chambers may increase in cold weather and decrease in warm weather because the system attempts to maintain a constant temperature at the wall sensors. Sensors have been moved off of the walls and closer to the vacuum chambers to mitigate this problem [78].

In addition to centralized temperature control systems, vibrations from local cooling, such as electronics cooling fans, can produce noise in DARM. We have mitigated acoustic coupling by placing electronics racks in separate rooms from other interferometer components, and by seismically isolating the electronics racks on elastic legs. Cooling systems for lasers etc. have also been problematic and have required additional seismic isolation.

5.3.4. Other site activities. Vehicle movements on sites have produced transients in DARM. This often occurs as vehicles cross bumps and gravel. Mitigation has included deeper burial of pipes that cross roads, removal of gravel from roads, patching of cracks and prohibition of travel in certain areas during observation. Even heavy steps in a control room can couple by producing beam jitter (see Section 5.1.1).

The springs isolating motor-driven equipment have been shorted (e.g. by drifts of blowing sand) or improperly installed. While vibration isolation of equipment is straightforward, acoustic isolation is often much more difficult and can "short circuit" well isolated systems. We have attempted to place sources of acoustic noise in separate rooms for this reason.

5.3.5. Humidity. Studies during the first two observing runs showed that periods of very low humidity inside of the buildings, associated with sub-freezing weather, are correlated with high glitch rates in DARM [79, 80]. One possible explanation is that reduced electrical conductivity associated with dry conditions can increase charge buildup and discharge in electronic systems such as piezo drivers.

\section{GW Event Validation}

In addition to investigating sources of environmental influences, knowledge acquired from environmental studies contributes to the vetting of GW event candidates. Analysis pipelines search the strain data for astrophysical signals. They are categorized into modeled searches for binary mergers that match the data to template waveforms (e.g. GstLAL [81] and PyCBC [82]) and unmodeled searches that identify excess energy coherent between multiple detectors (e.g. cWB [83], oLIB [84], and BW [85]).

Contamination of the GW data can occur through any of the means discussed in previous sections. Environmental noise has the potential to be correlated between detectors by stemming from a common source, such as through electromagnetic signals from distant sources or glitches in GPS-correlated electronics. The analysis pipelines 
estimate the false-alarm probabilities for GW events based on the background rate of randomly coincident events in the detector network. They generate background events by time-shifting the data stream of one detector relative to another by time steps much longer than the light travel time between detectors and longer than the duration of GW signals [86]. This method does not account for the possibility of transients being correlated between the detectors due to a common environmental source. Environmental noise is also particularly relevant to un-modeled searches. Unlike template-based methods, these searches make minimal assumptions about the signal waveform and rely more heavily on signal correlation between sites.

The first observation of a GW occurred on 14 Sept 2015 [3]. The event, a shortduration binary black hole merger designated GW150914, required a number of follow-up investigations to find potential noise sources around the time of the event [13]. This included an examination of the status of all PEM sensors and any significant signals they observed for possible contamination of the GW signal [87]. A few of the PEM sensors were not working, but because of redundancy, coverage was sufficient.

Comparisons between Q-transform spectrograms of all coincident events in environmental sensors to the time-frequency path of the event revealed that no environmental signals had paths similar to the event candidate. The signal-to-noise ratios of these signals were also compared to that of the event, showing that even if there were overlapping time-frequency paths, none of the environmental signals were large enough to influence the strain data at the SNR level of the event, based on multiplying the environmental signals by their respective sensor coupling functions.

The validation process for novel events such as GW150914 also includes redundant checks for global sources of environmental noise. We use a dedicated cosmic ray detector located below an input test mass at LHO to examine any association of cosmic ray showers to excess noise in DARM. We also check external observatories for coronal mass ejections, solar radio signals, geomagnetic signals, and RF signals in the detection band as well as higher frequencies.

There was specific concern over a co-incident extremely-high current $(504 \mathrm{kA})$ lightning strike over Burkina Faso, prompting additional studies of the effects of lightning on the interferometer [88]. Investigations of similar strikes found no effect on the strain data and investigations of closer strikes confirmed that the magnetometers were much more sensitive to lightning strikes than the interferometer was. In conclusion there was no reason to veto the first detection based on environmental disturbances.

Subsequent detections throughout O1 and and O2 employed a similar procedure; however the development of the method described in Section 3 for producing coupling functions for all sensors expedited the process. This was especially important for examining environmental noise during GW170817, the first long-duration event detected by LIGO $[5,89]$. The longer duration of this event $(75 \mathrm{~s})$ unsurprisingly overlapped with many environmental signals. Based on the coupling functions for those sensors, several of these environmental events were loud enough (estimated DARM signals of up to SNR 4) to have contributed to the interferometer readout, but not enough to account 
for the GW signal. Furthermore, none of them had a time-frequency morphology that correlated with any features in the candidate signal.

In O3, most of the procedure described above has been automated in order to handle the increase in detection rate. When an event is detected by the astrophysical search

pipelines, an Omega Scan [15] searches for transient noise in all PEM sensors in the time window spanning the event candidate and produces a Q-transform spectrogram of each sensor in which excess noise was detected, as well as the peak amplitude and frequency of the noise. The coupling function of each sensor is interpolated at the peak frequency and multiplied by the peak amplitude to estimate the contribution of the environmental noise to DARM. Sensors whose estimates exceed one tenth of the DARM background level are flagged for human input, requiring a comparison of the environmental signal morphology to that of the event candidate. If a there is sufficient signal overlap, reviewers may advise that analysts perform some noise removal in the data, such as by gating or filtering out the appropriate time or frequency range, before performing further follow up analyses. The event could be retracted, if gating or filtering out the environmental contribution would reduce the signal-to-noise ratio of the candidate to a level no longer consistent with a GW detection. During the first half of O3, no candidates were retracted on the basis of the environmental coupling check alone. Some human input was still required for all of the 39 events reported in Abbott et. al. (2020) [7], although little to no signal overlap of environmental transients was seen.

\section{Conclusions and Future Work}

Environmental disturbances continue to be a major topic of investigation in the current generation of gravitational wave detectors. With the transition from initial LIGO to aLIGO, the detectors underwent significant changes, many of which affected their sensitivity to environmental noise. The PEM system for monitoring these noise sources also saw modifications, to account for detector upgrades as well as to expand the coverage of the sensors. Over time we have developed new methods for tracking down noise sources as we have described here. We also described the method for quantifying environmental coupling and its limitations.

As $\mathrm{O} 4$ approaches, the detectors are undergoing further upgrades to improve their performance and begin the transition towards the "A+" phase [90]. These changes will introduce new hardware and infrastructure, such as a new $300 \mathrm{~m}$ filter cavity to implement frequency-dependent squeezing [91, 92]. The PEM system will continue to be expanded in order to monitor new noise sources that may arise with these upgrades. The installation of wall-mounted magnetic field injection coils will be completed at both sites ahead of $\mathrm{O} 4$ to provide full coverage of magnetic coupling. Additionally, shaker injections may also be incorporated in the weekly monitoring program to track changes in low-frequency vibrational coupling.

Further automation to the event validation process will be required to reduce the reliance on human input in future observing runs. This could include providing 
quantitative estimates on the overlap between the time-frequency path of a PEM signal and an event candidate, as well as estimating the DARM contribution at all times and frequencies rather than just at the time and frequency of the peak sensor amplitude. Environmental monitoring will continue to play a crucial role in the event validation as improved sensitivities bring about higher detection rates and the potential for novel sources of gravitational waves.

\section{Acknowledgements}

LIGO was constructed by the California Institute of Technology and Massachusetts Institute of Technology with funding from the National Science Foundation and operates under Cooperative Agreement No. PHY-1764464. Advanced LIGO was built under Grant No. PHY-0823459. The authors acknowledge support from NSF grants PHY1607336, PHY-1912604, PHY-1806656, and PHY-1806656. For this paper, we use the data from the Advanced LIGO detectors and we used the LIGO computing clusters to perform the analysis and calculations. 


\section{References}

[1] Aasi J et al. (LIGO Scientific Collaboration) 2015 Class. Quantum Grav. 32074001

[2] Martynov D V et al. 2016 Phys. Rev. D 93(11) 112004

[3] Abbott B P et al. (LIGO Scientific Collaboration and Virgo Collaboration) 2016 Phys. Rev. Lett. 116(6) 061102

[4] Abbott B P et al. (LIGO Scientific Collaboration and Virgo Collaboration) 2019 Phys. Rev. X 9(3) 031040

[5] Abbott B P et al. (LIGO Scientific Collaboration and Virgo Collaboration) 2017 Phys. Rev. Lett. 119(16) 161101

[6] Buikema A et al. 2020 Phys. Rev. D 102(6) 062003

[7] Abbott R et al. 2020 (Preprint arXiv:2010.14527)

[8] Effler A, Schofield R M S, Frolov V V, González G, Kawabe K, Smith J R, Birch J and McCarthy R 2015 Class. Quantum Grav. 32035017

[9] Fiori I et al. 2020 Galaxies 882

[10] Davis D et al. (LIGO Scientific Collaboration and Virgo Collaboration) 2020 in progress Class. Quantum Grav.

[11] Nuttall L K et al. 2015 Class. Quantum Grav. 32245005

[12] Nuttall L K 2018 Philos. Trans. R. Soc. A 37620170286

[13] Abbott B P et al. (LIGO Scientific Collaboration and Virgo Collaboration) 2016 Class. Quantum Grav. 33134001

[14] Davis D, Massinger T, Lundgren A, Driggers J C, Urban A L and Nuttall L 2019 Class. Quantum Grav. 36055011

[15] Chatterji S, Blackburn L, Martin G and Katsavounidis E 2004 Class. Quantum Grav. 21 S1809S1818

[16] Zevin M et al. 2017 Class. Quantum Grav. 34064003

[17] Bahaadini S, Noroozi V, Rohani N, Coughlin S, Zevin M, Smith J, Kalogera V and Katsaggelos A 2018 Information Sciences 444 172-186

[18] Smith J R, Abbott T, Hirose E, Leroy N, MacLeod D, McIver J, Saulson P and Shawhan P 2011 Class. Quantum Grav. 28235005

[19] Robertson N A et al. 2002 Class. Quantum Grav. 19 4043-4058

[20] Aston S M et al. 2012 Class. Quantum Grav. 29235004

[21] Schofield R M S 2011 Early work to reduce aLIGO environmental coupling https://dcc.ligo. org/LIGO-G1101046/public

[22] Schofield R M S 2010 Why LIGO's range is limited by ground motion: the likely source of upconversion https://dcc.ligo.org/LIGO-G1000923/public

[23] PEM Central http://pem.ligo.org

[24] Schofield R M S 2012 aLIGO PEM System Upgrade https://dcc.ligo.org/LIGO-T1200221/ public

[25] Bartington Instruments Mag-03 https://www.bartington.com/mag-03/

[26] Covas P B et al. (LSC Instrument Authors) 2018 Phys. Rev. D 97(8) 082002

[27] Hardwick T and Schofield R M S 2013 Magnetometer tripod mount prototype tested https: //alog.ligo-wa.caltech . edu/aLOG/index . php?callRep=7456

[28] LEMI LLC 2016 LEMI-120 https://lemisensors. com/?p=245

[29] Thrane E, Christensen N and Schofield R M S 2013 Phys. Rev. D 87123009

[30] Thrane E, Christensen N, Schofield R M S and Effler A 2014 Phys. Rev. D 90023013

[31] Coughlin M W et al. 2018 Phys. Rev. D 97(10) 102007

[32] Abbott R, Torrie C, Robertson N, Sanchez E, Sanchez L, Weiss R, Fritschel P and Coyne D 2018 Electric field meter final design document https://dcc.ligo.org/LIGO-E1800050/public

[33] Mansell G, Schofield R M S, Weiss R, Cahillane C and Abbott R 2015 Electric Field Meter testing with viewport-mounted capacitor https://alog.ligo-wa.caltech.edu/aLOG/index. 
php? callRep $=41559$

[34] PASSCAL Instrument Center Guralp CMG-3T Broadband Sensor https://www.passcal.nmt. edu/content/instrumentation/sensors/broadband-sensors/cmg-3t-bb-sensor

[35] Peterson J R 1993 Observations and modeling of seismic background noise URL https://doi. org/10.3133/ofr93322

[36] Wilcoxon Sensing Technologies Compact seismic accelerometer 731-207 https://buy.wilcoxon. $\mathrm{com} / 731-207 . \mathrm{html}$

[37] Brüel \& Kjær 1/2" Condenser Microphone Cartridge Type 4130 https://www.bksv.com/media/ $\mathrm{doc} / \mathrm{Bp0020}$.pdf

[38] Brüel \& Kjær The Falcon Range 1/2" Microphones - Types 4188 to 4193 https://www. bksv . $\mathrm{com} / \mathrm{media} / \mathrm{doc} / \mathrm{Bp} 1380 . \mathrm{pdf}$

[39] Brüel \& Kjær Battery-powered CCLD Signal Conditioners https://www.bksv.com/-/media/ literature/Product-Data/bp2387.ashx

[40] Universal Radio Inc AOR AR5000A Wideband Receiver https://www.universal-radio.com/ catalog/widerxvr/3705spec.html

[41] Kruk J and Schofield R M S 2016 Environmental Monitoring: Coupling Function Calculator https://dcc.ligo.org/LIGO-T1600387/public

[42] Nguyen P 2020 PEM Coupling Function Tools https://git.ligo.org/philippe.nguyen/ pemcoupling

[43] Washimi T, Yokozawa T, Tanaka T, Itoh Y, Kume J and Yokoyama J 2020 (Preprint arXiv: 2010.14527)

[44] Nguyen P, Effler A and Schofield R M S 2021 PEM coupling function uncertainty estimation https : //alog.ligo-wa. caltech.edu/aLOG/index . php?callRep=57672

[45] Nguyen P and Schofield R M S 2019 Coupling estimates from PEM injections correctly predict LLO DARM glitch near S190510g from thunder-driven vibration at EY https://alog.ligo-la. caltech $\cdot$ edu/aLOG/index $\cdot$ php? callRep $=46025$

[46] Nguyen P and Schofield R M S 2020 Coupling estimates of helicopter flyovers at both sites during O3 https://alog. ligo-wa. caltech. edu/aLOG/index $\cdot$ php?callRep=56285

[47] Banagiri S, Covas P and Schofield R M S 2019 PEM with Rain using HAM6 Vac accelerometer https: //alog. ligo-wa. caltech .edu/aLOG/index . php?callRep=49495

[48] Schofield R M S, Nguyen P, Banagiri S, Merfeld K and Effler A 2019 HVAC noise in DARM roughly consistent with prediction for septum from PEM injections https://alog.ligo-wa. caltech $\cdot$ edu/aLOG/index $\cdot$ php? callRep $=48912$

[49] APS Dynamics APS 113 ELECTRO-SEIS Long Stroke Shaker https://apsdynamics.com/en/ products/vibration-exciter/ball-bearing-exciter.html

[50] Piezosystems Piezo nanopositioning devices, actuators \& controllers https://www.piezosystem. $\mathrm{com} /$

[51] Brüel \& Kjær Sound and vibration measurement https://www.bksv.com/

[52] Schofield R M S, Ball M, Helmling-Cornell A, Shoemaker D, Vorvick C and Banagiri S 2019 New beating-shaker technique localizes coupling site of $48 \mathrm{~Hz}$ DARM peak, and black glass eliminates it https: //alog. ligo-wa. caltech $\cdot$ edu/aLOG/index $\cdot$ php? callRep=52184

[53] Schofield R M S, Rodruck M, Punken O and Savage R 2012 PSL table vibration from chiller solved by removing quick-connects https://alog.ligo-wa.caltech.edu/aLOG/index.php?callRep= 4945

[54] Schofield R M S and Vorvick C 2019 An easy optic damping system reduces remaining optic mount jitter peaks in DARM by more than 3 https://alog.ligo-wa.caltech.edu/aLOG/index.php? callRep $=46494$

[55] Schofield R M S 2019 PSL periscope and optic mount resonance tuning https://alog.ligo-wa. caltech $\cdot$ edu/aLOG/index $\cdot$ php? callRep $=18417$

[56] Viton ${ }^{\mathrm{TM}}$ High-Performance Fluoroelastomers https://www.viton.com

[57] Schofield R M S, Izumi K and Effler A 2016 HAM6 damping reduced linear and non-linear coupling 
by 3, proposed OMC dither settings would reduce non-linear coupling by a further factor of 8 https://alog.ligo-wa.caltech.edu/aLOG/index . php?callRep=30380

[58] Schofield R M S and Radkins H 2015 Tilt noise is much smaller $40 \mathrm{~m}$ from building, coherence still high below $0.5 \mathrm{~Hz}$ https://alog.ligo-wa. caltech. edu/aLOG/index.php?callRep=19210

[59] Schofield R M S and Radkins H $201617 \mathrm{mph}$ wind-induced low-f tilt and vibration coherence less than 0.5 at $5 \mathrm{~m}$ at EY https://alog. ligo-wa. caltech. edu/aLOG/index . php?callRep=27170

[60] Venkateswara K, Hagedorn C A, Turner M D, Arp T and Gundlach J H 2014 Rev. Sci. Instrum. 85015005

[61] Venkateswara K et al. 2017 Bull. Seismol. Soc. Am. 107 709-717

[62] Lantz B and Radkins H 2020 Impact of the wind fences https://dcc.ligo.org/LIGO-G2000112/ public

[63] Soni S et al. 2020 Class. Quantum Grav. 38025016

[64] Schofield R M S, Covas P, Effler A and Savage R 2017 Why the GW channel detects thirsty black ravens along with colliding black holes https://alog.ligo-wa.caltech.edu/aLOG/index. php? callRep $=37630$

[65] Schofield R M S 2017 The Swiss Cheese baffle (MCA1) is a dominant range-reducing source of vibration coupling to DARM, and a proposal for mitigation https://alog.ligo-wa.caltech . edu/aLOG/index $\cdot$ php? callRep $=35735$

[66] Schofield R M S 2017 Swiss cheese baffle damping gave 5 reduction in maximum scattering shelf frequency and decay time https://alog.ligo-wa.caltech.edu/aLOG/index.php?callRep= 36979

[67] Effler A 2020 EY scatter-inducing resonance appears to be around 3.7 Hz https://alog. ligo-la. caltech $\cdot$ edu/aLOG/index $\cdot$ php? callRep $=53025$

[68] Schofield R M S 2020 ETMY Manifold/Cryopump Baffle barrel resonates at the $3.8 \mathrm{~Hz}$ frequency of scattering noise; three LHO baffles need damping, ETMY, ITMY, and ETMX https: //alog.ligo-wa.caltech.edu/aLOG/index.php?callRep=56857

[69] Effler A 2020 PEM sweeps cannot find the cryo baffle resonances! https://alog.ligo-la. caltech $\cdot$ edu/aLOG/index $\cdot$ php? callRep=54336

[70] Schofield R M S et al. 2020 Scattering noise up to $100 \mathrm{~Hz}$ from $0.1 \mathrm{~Hz}$ relative motion of test and reaction masses at LHO and LLO, and potential mitigation https://alog.ligo-wa.caltech. edu/aLOG/index $\cdot$ php?callRep $=54298$

[71] Schofield R M S 2018 Recent views from beam spots, and a first attempt at ranking the observed glints for wide-angle scattering noise. https://alog.ligo-wa.caltech.edu/aLOG/index.php? callRep $=41142$

[72] Schofield R M S 2013 Investigations of magnetic coupling to the quads https://dcc.ligo.org/ LIGO-G1300300/public

[73] Schofield R M S 2015 Beam tube particulate injection https://alog.ligo-wa.caltech.edu/ aLOG/index $\cdot$ php?call Rep $=20354$

[74] Schofield R M S 2016 First look at drop in particulate glitch probability with lower tap amplitude and a simple microphone-based beam-tube monitoring scheme https://alog. ligo-wa . caltech $\cdot$ edu/aLOG/index $\cdot$ php? callRep $=25962$

[75] Palamos J 2015 PEM injection report - RF coupling https://alog.ligo-wa.caltech.edu/aLOG/ index $\cdot$ php? callRep $=23252$

[76] Palamos J 2020 Search for gravitational waves associated with gamma-ray bursts during LIGO's second observing run https://dcc.ligo.org/LIGO-P2000352/public

[77] Palamos J, Schofield R M S and Dwyer S 2015 Evidence that mystery $650 \mathrm{~Hz}$ noise caused by COMM VCO https://alog.ligo-wa.caltech.edu/aLOG/index . php?callRep=24062

[78] Schofield R M S 2017 Set the temperature lower when it's cold outside? https://alog.ligo-wa. caltech $\cdot$ edu/aLOG/index $\cdot$ php? callRep $=33320$

[79] Schale P, Palamos J and Schofield R M S 2016 A class of O1 blip glitches is correlated with low relative humidity in the buildings https://alog.ligo-wa.caltech.edu/aLOG/index.php? 


\section{callRep $=28534$}

[80] Cabero M et al. 2019 Class. Quantum Grav. 36155010

[81] Cannon K et al. 2012 ApJ $\mathbf{7 4 8} 136$

[82] Usman S et al. 2016 Class. Quantum Grav. 33215004

[83] Klimenko S, Yakushin I, Mercer A and Mitselmakher G 2008 Class. Quantum Grav. 25114029

[84] Lynch R, Vitale S, Essick R, Katsavounidis E and Robinet F 2017 Phys. Rev. D 95(10) 104046

[85] Cornish N J and Littenberg T B 2015 Class. Quantum Grav. 32135012

[86] Wąs M, Bizouard M, Brisson V, Cavalier F, Davier M, Hello P, Leroy N, Robinet F and Vavoulidis M 2009 Class. Quantum Grav. 27015005

[87] Schofield R M S, Roma V, Palamos J, Hardwick T, Effler A, Frey R and Talukder D 2018 PEM vetting report for GW150914 https://dcc.ligo.org/LIGO-T1800505/public

[88] Schofield R M S 2018 Lightning, LIGO, and GW150914 https://dcc.ligo.org/LIGO-T1800506/ public

[89] Schofield R M S, Nguyen P, Roma V, Palamos J, Schale P, Effler A, Talukder D and Frey R 2018 PEM vetting report for GW170817 https://dcc.ligo.org/LIGO-T1800508/public

[90] Miller J, Barsotti L, Vitale S, Fritschel P, Evans M and Sigg D 2015 Phys. Rev. D 91(6) 062005

[91] Evans M, Barsotti L, Kwee P, Harms J and Miao H 2013 Phys. Rev. D 88(2) 022002

[92] McCuller L, Whittle C, Ganapathy D, Komori K, Tse M, Fernandez-Galiana A, Barsotti L, Fritschel P, MacInnis M, Matichard F, Mason K, Mavalvala N, Mittleman R, Yu H, Zucker M E and Evans M 2020 Phys. Rev. Lett. 124(17) 171102 